\title{
The Corridor Problem: Preliminary Results on the No-toll Equilibrium
}

\author{
Richard Arnott \\ Department of Economics, University of California, Riverside \\ Riverside, CA 92506, richard.arnott@ucr.edu, (951)827-1581 \\ Elijah DePalma \\ Department of Statistics, University of California, Riverside \\ Riverside, CA 92506, edepalma@math.ucr.edu, (951)234-2190
}

January 13, 2010

\begin{abstract}
Consider a traffic corridor that connects a continuum of residential locations to a point central business district, and that is subject to flow congestion. The population density function along the corridor is exogenous, and except for location vehicles are identical. All vehicles travel along the corridor from home to work in the morning rush hour, and have the same work start time but may arrive early. The two components of costs are travel time costs and schedule delay (time early) costs. Determining equilibrium and optimum traffic flow patterns for this continuous model, and possible extensions, is termed "The Corridor Problem". Equilibria must satisfy the trip-timing condition, that at each location no vehicle can experience a lower trip price by departing at a different time. This paper investigates the no-toll equilibrium of the basic Corridor Problem.
\end{abstract}

Keywords: morning commute; congestion; corridor; equilibrium

\section{Introduction $^{1}$}

In recent years, considerable work has been done examining the equilibrium dynamics of rush-hour traffic congestion. The central feature is the trip-timing condition, that no vehicle

\footnotetext{
${ }^{1}$ Arnott would like to thank seminar participants at U.B.C., Dong Hua University, Kyoto University, the University of Tokyo, and the Free University of Amsterdam, as well as session participants at the 2004 and 2008 AEA Meetings for helpful remarks. Yasushi Atsumi, Malachy Carey, Marvin Kraus, Stephen Ross, and above all Ian Sue Wing, provided especially useful comments, either at presentations or on early versions of the paper. He would like to thank Danqing Hu, Junling Wang, Xiaomeng Yang and Li Zeng for excellent research assistance.
} 
can experience a lower trip price by departing at a different time, where trip price includes the cost of travel time, the cost of traveling at an inconvenient time (termed schedule delay cost), and the toll, if applicable. The theoretical work on the topic has been in the context of Vickrey's model of a deterministic queue behind a single bottleneck (Vickrey (1969)), with some papers treating extensions to very simple networks, with each link containing a bottleneck.

While insightful, the work does not provide much insight into the spatial dynamics of rush-hour traffic congestion. Start by visualizing a departure rate surface over a metropolitan area. What does it look like at a point in time, and how does it change over the rush hour? Similarly, what do the flow, density, and velocity surfaces look like, and how do they evolve?

This paper takes a modest step forward in examining the spatial equilibrium dynamics of rush-hour congestion. It lays out perhaps the simplest possible model with continuous time and space that can address the issue. The metropolitan area is modeled as a single traffic corridor of uniform width joining the suburbs to the central business district (CBD), a point in space; the population entering each point along the corridor over the rush hour is taken as given; except for their locations, vehicles are identical, having a common work start time at the CBD and a common trip price function that is linear in travel time and schedule delay; congestion takes the form of classic flow congestion; and there is no toll. The paper poses the simple question: What pattern(s) of departures satisfy the trip-timing condition? We term the corresponding problem and extensions, including determination of socially optimal allocations, "The Corridor Problem".

Unless some insight has eluded us, answering this question in the context of even so basic a model is surprisingly difficult (but if it were not difficult, it would likely have been solved). We have not yet succeeded in obtaining a complete solution, but because of the problem's difficulty feel justified in reporting on what progress we have made.

There are good reasons to believe that the Corridor Problem is important. On the practical side, solving the problem would provide a point of entry to understanding the spatial dynamics of rush-hour traffic congestion, which is surely important in the enlightened design of road and mass transit networks. On the theoretical side, the problem has posed a stumbling block to the development of three lines of theoretical literature on the economics of traffic congestion. During the 1970s several papers were written on the economics of traffic congestion in the context of the monocentric city and related models (Solow and Vickrey (1971); Solow (1972); Kanemoto (1976); and Arnott (1979)), assuming that traffic flow is constant over the day. Their focus was on second-best issues, in particular on how the underpricing of urban auto congestion distorts land use and affects capacity investment rules. Are the insights from that literature substantially modified when account is taken of the ebb and flow of traffic? At around the same time, Beckmann and Puu (e.g., Beckmann and Puu (1985)) started work on two-dimensional, steady-state continuous flow models of traffic congestion. Solving the Corridor Problem might provide insight into how to extend their work to non-stationary traffic flow. In the late 1980s, Arnott, dePalma and Lindsey (1994) attempted to generalize the bottleneck model to a traffic corridor, modeled as a series of bottlenecks with entry points between them. Because of the model's linearity, the solution degenerated into the treatment of multiple cases, the number rising geometrically with the number of bottlenecks. Thus, despite its elegant simplicity in other contexts, the bottleneck model does not appear well suited to examining the spatial dynamics of traffic congestion. 
There is some prior work on the equilibrium spatial dynamics of urban traffic congestion. In the context of the monocentric model, Yinger (1993) assumed that vehicles at the urban boundary are the first to depart and depart together, and are followed by successive cohorts from increasingly more central locations, and solved for the implied spatial dynamics of congestion over the rush hour. Ross and Yinger (2000) proved that the departure pattern assumed in Yinger (1993) does not satisfy the trip-timing condition, and that no other simple departure pattern does either. In earlier work, Arnott (2004) conjectured an equilibrium departure pattern but, since he was unable to prove his conjecture, investigated a discretized variant of the problem, with buses and bus stops, termed the "bus-corridor problem". Congestion takes the form of bus speed varying inversely with the number of passengers. The numerical examples of the bus-corridor problem presented there are consistent with the form of departure set conjectured for the corridor problem proper, but do not prove the conjecture since the discretization alters the problem. Tian, Huang and Yang (2007) derive the equilibrium properties of a variant of the bus-corridor problem in which congestion takes the form of crowding costs that increase in the number of passengers, provide some solution algorithms, and present numerical examples. The numerical examples of this variant of the bus-corridor problem are also consistent with the form of the departure set conjectured for the Corridor Problem proper in Arnott (2004), but again do not prove the conjecture because the problem is somewhat different.

Section 2 presents the basic model and states the problem. Section 3 derives some implications of the trip-timing condition. Section 4 states the heuristic reasoning underlying an initial proposed solution. Section 5 undertakes the mathematical analysis of the initial proposed solution, in the process demonstrates that the initial proposed solution is not consistent in one respect with the trip-timing condition, and modifies the proposed solution. Section 6 develops an algorithm to solve numerically for a departure pattern consistent with the modified proposed solution. Section 7 presents the results of the numerical algorithm. Section 8 takes stock, and conjectures how the proposed solution needs to be further modified to obtain a full solution to the Corridor Problem.

\section{Model Description}

Consider a traffic corridor of constant width that connects a continuum of residential locations, "the suburbs," to a point central business district (CBD) that lies at the eastern end of the corridor, as shown in Figure 1. Location is indexed by $x$, the distance from the outer boundary of the residential settlement towards the CBD, which is located at $\bar{x}$. $N(x) d x$ denotes the exogeneous number of vehicles departing between $x$ and $x+d x$ over the rush hour. It is assumed that $N(x)$ is strictly positive for $x \in(0, \bar{x})$.

\subsection{Trip Cost}

Each morning all vehicles travel from their departure location to the CBD and have the common desired arrival time, $\bar{t}$. Late arrivals are not permitted, and in the absence of a toll the common travel cost function is

$$
C=\alpha(\text { travel time })+\beta(\text { time early }),
$$




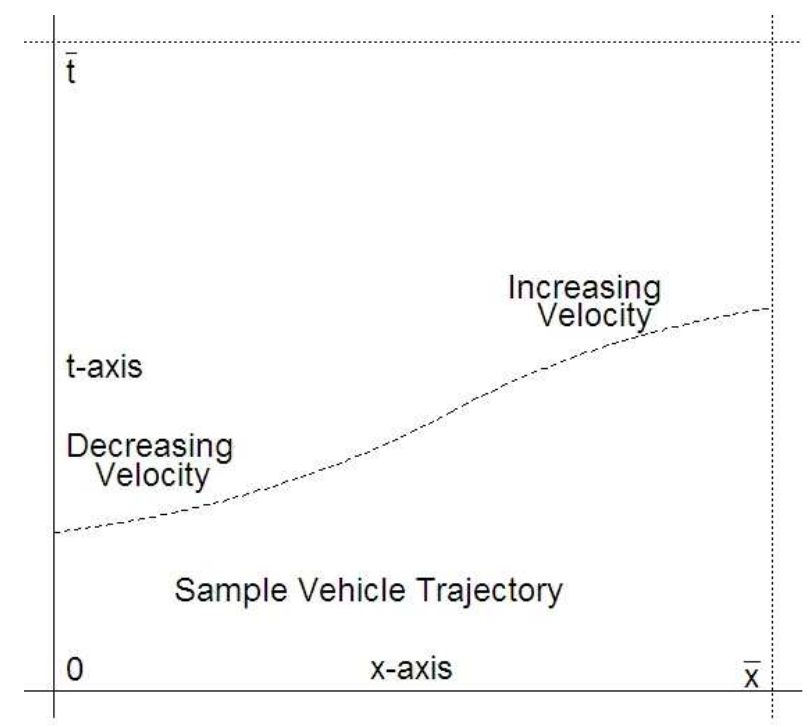

Figure 1: Traffic Corridor, Space-time Diagram

where $\alpha$ is the value or cost of travel time, and $\beta$ is the value or cost of time early. It is assumed that $\alpha>\beta$, which is supported by empirical evidence (Small (1982)). Let $T(x, t)$ denote the travel time of a vehicle that departs from $x$ at time $t$. Then $t+T(x, t)$ is the vehicle's arrival time, so that $\bar{t}-[t+T(x, t)]$ is its time early, so that the trip cost may be written as

$$
C(x, t)=\alpha T(x, t)+\beta(\bar{t}-[t+T(x, t)]) .
$$

There are no tolls, so that, at each time and location, trip price equals trip cost.

\subsection{Continuity Equation}

Classical flow congestion is assumed, which combines the equation of continuity with an assumed relationship between density and flow. Recall that we have assumed the road to be of constant width. Accordingly, at location $x$ and time $t$, let $\rho(x, t)$ denote the density of vehicles per unit length, and let $v(x, t)$ denote velocity. The relationship between velocity and density is written as

$$
v(x, t)=V(\rho(x, t)),
$$

with $V^{\prime}<0$. It is typically assumed that: i) $V$ steadily decreases with $\rho$, so that flow, $F=\rho V$, is a smooth convex function of $\rho$; ii) flow is zero with zero density and also with jam density. The equation of continuity is simply a statement of conservation of mass, that the change in the number of vehicles on a section of road of infinitesimal length equals the inflow minus the outflow. Letting $n(x, t) \geq 0$ denote the entry rate onto the road, the equation of continuity is

$$
\frac{\partial \rho}{\partial t}+\frac{\partial}{\partial x}(\rho V)=n(x, t) .
$$




\subsection{Trip-Timing Condition (TT)}

There are two equilibrium conditions. The first is that everyone commute. If we let $\mathcal{D}$ denote the departure set, i.e., the set of $(x, t)$ points for which departures occur in equilibrium, then the condition that everyone commute can be written as

$$
\int_{(x, t) \in \mathcal{D}} n(x, t) d t=N(x) \quad \forall x \in[0, \bar{x}] .
$$

The second equilibrium condition is the trip-timing condition (TT), that no vehicle can experience a lower trip price by departing at a different time. Letting $p(x)$ denote the equilibrium trip price at location $x$, the TT condition can be written as

$$
\begin{aligned}
& C(x, t)=p(x) \quad \forall(x, t) \in \mathcal{D} \quad \text { (Equality Component of the TT) } \\
& C(x, t) \geq p(x) \quad \forall(x, t) \notin \mathcal{D} \quad \text { (Inequality Component of the TT) }
\end{aligned}
$$

which states that at no location can the trip price be reduced by traveling outside the departure set at that location. A no-toll equilibrium is a departure pattern, $n(x, t) \geq 0$, and a trip price function, $p(x)$, such that the equilibrium conditions are satsified, with $T(x, t)$ obtained from the solution to the continuity equation.

\section{Implications of the Trip-Timing Condition}

\subsection{Relation between Arrival and Departure Times}

From (1) and (3),

$$
T(x, t)=\frac{p(x)-\beta(\bar{t}-t)}{\alpha-\beta} \quad \forall(x, t) \in \mathcal{D}
$$

Hence, over the departure set at each location, travel time increases linearly in the departure time at the rate $\frac{\beta}{\alpha-\beta}$. In particular, if two vehicles leave the same location, $x$, within the departure set, separated by time $\Delta t$, then the difference between their arrival times is $\Delta a$, where

$$
\Delta a=\frac{\alpha}{\alpha-\beta} \Delta t
$$

Suppose, for the sake of argument, that the interior of the departure set is non-empty and connected. The only way for (4) to be satisfied is for cohorts of vehicles to be identical, except for the entry of vehicles closer to the CBD for later cohorts. To see this intuitively, employ the bus-corridor discretization of the problem, for which the speed of a bus is related to its number of passengers.

Suppose that the first bus to depart picks up passengers at stops 1 and 2, and that the second bus to depart picks up passengers at stops 1, 2, and 3. The trip-timing equilibrium condition requires that travel time on the second bus be higher than travel time on the first bus by the same amount for passengers boarding at stop 1 as for those boarding at the stop 2. The travel time increase for those boarding at stop 1 equals the travel time increase between stops 1 and 2, 2 and 3, - . The travel time increase for those boarding at stop 2 equals the travel time increase between stops 2 and $3,-$. For these travel time increases to 
be the same requires that the travel time between stops 1 and 2 be the same for the first and second buses, which requires that at bus stop 1 the same number of passengers board the first and second buses. The argument in the next section formalizes this intuitive argument.

\subsection{Constant Departure Rate within Interior of Departure Set}

It will prove convenient at this point to make the transformation of variables

$$
a(x, t)=t+T(x, t)
$$

where $a(x, t)$ is the arrival time at the CBD of a vehicle that departs location $x$ at time $t$. If $\hat{T}(x, a)$ is the travel time of a vehicle that arrives at the CBD at time $a$, then the inverse transformation is

$$
t(x, a)=a-\hat{T}(x, a)
$$

which relates departure time to arrival time. The trip-timing condition, expressed in terms of arrival time, is

$$
p(x)=\alpha \hat{T}(x, a)+\beta(\bar{t}-a) \quad \forall(x, a) \in \mathcal{A}
$$

where, $\mathcal{A}$, the arrival set, is the set of all $(x, a)$ for which the arrival rate is positive. The advantage of working in terms of arrival time is that $\hat{T}(x, a)$ tracks the cohort of vehicles that arrives at time $a$. Since a vehicle with arrival time $a$ passes location $x$ at time $a-\hat{T}(x, a)$,

$$
\hat{T}(x, a)=\hat{T}(x+d x, a)+\frac{d x}{v(x, a-\hat{T}(x, a))}
$$

and so

$$
\hat{T}_{x}(x, a)=-\frac{1}{v(x, a-\hat{T}(x, a))}=-\frac{1}{V(\rho(x, a-\hat{T}(x, a)))}
$$

Differentiation of (5) with respect to $a$ and then $x$ yields

$$
\begin{gathered}
\hat{T}_{a}=\frac{\beta}{\alpha} \quad \forall(x, a) \in \operatorname{int}(\mathcal{A}) \\
\hat{T}_{a x}=0 \quad \forall(x, a) \in \operatorname{int}(\mathcal{A}),
\end{gathered}
$$

while differentiation of (6) with respect to $a$ yields

$$
\hat{T}_{x a}(x, a)=\frac{V^{\prime} \rho_{t}\left(1-\hat{T}_{a}\right)}{V^{2}}
$$

From equality of mixed partial derivatives, it follows that the right-hand side of (7) equals zero, and since $V^{\prime}, 1-\hat{T}_{a}$ and $V$ are all strictly nonzero, it follows that

$$
\rho_{t}(x, a-\hat{T}(x, a))=0 \quad \forall(x, a) \in \operatorname{int}(\mathcal{A})
$$

or,

$$
\rho_{t}(x, t)=0 \quad \forall(x, t) \in \operatorname{int}(\mathcal{D}) .
$$


(8) states that traffic density is constant at a particular location over the interior of the departure set at that location. Since $\rho_{t}=0$, the continuity equation reduces to

$$
\frac{\partial}{\partial x}(\rho V(\rho))=n(x, t)
$$

Differentiating this equation with respect to $t$ yields

$$
\frac{\partial n}{\partial t}=\frac{\partial}{\partial t}(\rho V)_{x}=\frac{\partial}{\partial x}(\rho V)_{t}=\frac{\partial}{\partial x} 0=0
$$

Thus, at each location, the departure rate is constant over the interior of the departure set,

$$
n(x, t)=n(x) \quad \forall(x, t) \in \operatorname{int}(\mathcal{D}) .
$$

\section{Proposed Departure Set}

Consider two vehicle trajectory segments, running from some $x^{\prime}$ to $x^{\prime \prime}$, both of which are in the interior of the departure set, an earlier one and a later one. For each $x$, traffic density is the same for both trajectories (8), as is the departure rate (9). Furthermore, at all locations between $x^{\prime}$ and $x^{\prime \prime}$ the travel time of the later trajectory exceeds the travel time of the earlier trajectory by the same amount. This requires that travel time between $x^{\prime \prime}$ and the CBD be higher for the later trajectory, which in turn requires that more vehicles enter the road between $x^{\prime \prime}$ and the CBD for the later trajectory. One way this can be achieved is for the first departure time at each location to be later for more central locations. Put alternatively, later trajectories pick up vehicles at increasingly central locations.

Figure 2 displays a departure set consistent with this reasoning. Time is renormalized so that $t=0$ corresponds to the start of the morning commute (at $x=0$.) The departure set is connected. The lower boundary gives the time of the first departure at each location, and the upper boundary the time of the last departure at each location. A sample trajectory is shown as the dashed line. The first trajectory contains vehicles from only the most distant location. Succeeding trajectories contain vehicles from locations successively closer to the CBD, as well as from all more distant locations. The last trajectory, which arrives at the CBD exactly at the desired arrival time, $\bar{t}$, contains vehicles from all locations. We refer to the departure set as Region I, and the region below the departure set as Region II.

Since the pattern of density by location in the interior of the departure set does not change over time, at any location the number of vehicles entering at more distant locations must equal the flow at that location. At more central locations therefore, within the departure set the flow rate must be higher, which is inconsistent with hypercongestion. Thus, along a vehicle trajectory velocity decreases from $x=0$ to the lower boundary of the departure set, and then speeds up from the lower boundary of the departure set to the CBD because no vehicles enter the road at those locations. Thus, a vehicle trajectory is convex in the interior of Region I and concave in the interior of Region II.

The above line of reasoning leaves open the properties of the boundary of the departure set. The rest of this section will sketch a more formal derivation of the properties of the departure set. 


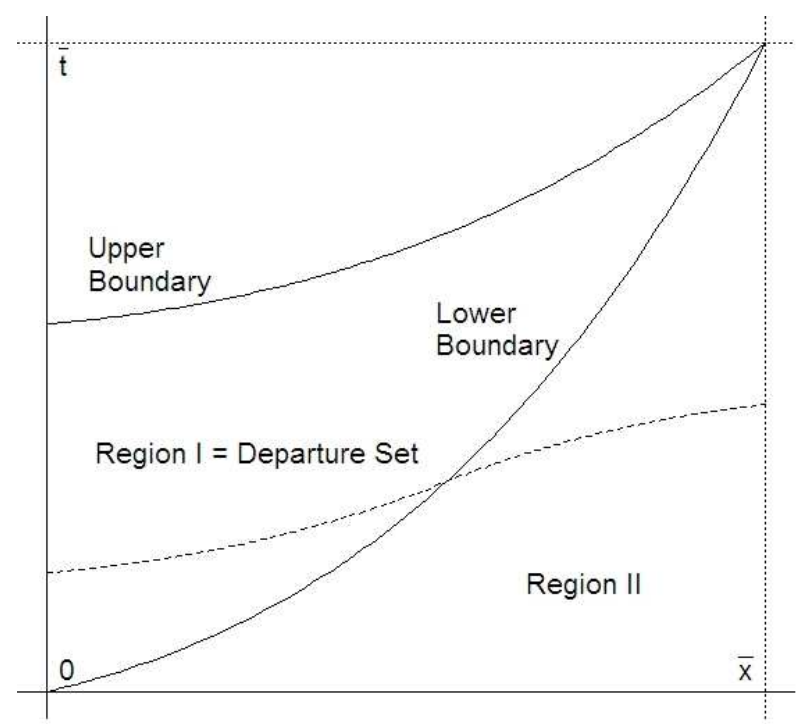

Figure 2: Horn-shaped proposed departure set. Upper boundary is a vehicle trajectory corresponding to the final cohort of vehicles to arrive at the CBD. Dashed line indicates a sample vehicle trajectory, with decreasing velocity within the departure set (Region I) and increasing velocity outside the departure set (Region II). Note that the slope of the dashed line equals the slope of the upper boundary, up to the point of leaving the departure set.

\subsection{General Properties of the Departure Set}

We shall argue that the departure set has the following properties:

Property 1 The upper boundary of the departure set is a vehicle trajectory.

Property 2 At any location, the departure rate on the upper boundary of the departure set is the same as in the interior of the departure set.

Property 3 At any location, the departure set at that location is a connected set.

Property 4 The departure set is connected and does not contain holes.

Property 5 Mass points on the lower boundary of the departure set are not inconsistent with the trip-timing condition, and cannot be ruled out.

\section{Property 1: Upper Boundary of the Departure Set Is a Vehicle Trajectory.}

If the upper boundary of the departure set is a vehicle trajectory, the trajectory must arrive at the CBD exactly at $\bar{t}$. Suppose not, and that the trajectory arrives at the CBD at $t^{\prime}<\bar{t}$. Then at any location there is a departure time for which a vehicle can depart, experience no traffic congestion, and arrive at the CBD between $t^{\prime}$ and $\bar{t}$, experience less travel time and arrive less early than all other vehicles departing from that location, which is inconsistent with the trip timing condition. 
Suppose that the final cohort of vehicles to arrive at the CBD does not contain vehicles that depart from $x=0$, and that the latest departure from $x=0$ in the departure interval is at $t^{\prime}$. Then a vehicle departing $x=0$ slightly after $t^{\prime}$ can travel at free-flow travel speed until it meets the cohort of vehicles that departs from $x=0$ at $t^{\prime}$, hence experiencing a lower trip cost than the vehicle that departs $x=0$ at $t^{\prime}$.

With some modification, the same line of reasoning can be applied to establish that the final cohort must contain vehicles from every location.

\section{Property 2: At any Location, the Departure Rate on the Upper Boundary of the Departure Set Is the Same as in the Interior.}

(8) indicates that, at each location, density and hence velocity is constant in the interior of the departure set. Hence, in the interior of the departure set velocity can be written as $v(x)$ and density can be written as $\rho(x)$. If the departure rate were different on the upper boundary (including the possibility of mass points), then the velocity as a function of location for the last cohort would not be $v(x)$, which can be shown to imply violation of the trip-timing condition.

\section{Property 3: At Any Location, the Departure Set at that Location Is a Connected Set}

We have proved that, at a particular location in the interior of the departure set, density (8) and the entry rate (9) must be constant. Now suppose that the departure set at location $x^{\prime}$ is disconnected. Then a vehicle departing from $x^{\prime}$ outside of the departure set will experience a lower traffic density, and hence a higher velocity, than a vehicle departing within the departure set at the same location. Therefore, to a vehicle that departs $x^{\prime}$ inside of the departure set at an earlier time, a vehicle that departs $x^{\prime}$ outside the departure set at a later time will incur less travel time cost up to the point when it either enters the departure set (i.e., joins a cohort of vehicles that depart within the departure set) or reaches the CBD. This is inconsistent with the TT-condition, which requires that vehicles that depart the same location but at a later time incur greater travel time cost.

\section{Property 4: The Departure Set Is Connected and Does Not Contain Holes.}

Property 4 easily follows from Properties 1 and 3, and the requirement that the population density be nonzero at all locations up to the edge of the metropolitan area. Since the population density is nonzero at all locations the departure set is nonempty at all locations. From Property 3 the departure set at a given location is a connected set. From Property 1 the upper boundary of the departure set is a vehicle trajectory, which is a connected set. Thus, the departure set is the union of connected sets, each of which has nonempty intersection with a connected set, and is therefore connected. 


\section{Property 5: Mass Points on the Lower Boundary of the Departure Set Cannot Be Ruled Out.}

Mass points along the lower boundary of the departure set cause discontinuities in the flow (shocks) as vehicles leave the departure set. If these mass points are sufficiently small that their range of influence does not extend into the interior of the departure set ${ }^{2}$, then the analysis of the traffic flow within the departure set is unchanged. The distribution of mass points is such that the TT-condition is satisfied.

\subsection{Proposed Departure Set}

In his earlier work on the Corridor Problem, Arnott (2004) had established Properties 1 and 2 , and conjectured Properties 3 and 4 . He did not, however, consider the possibility of mass points on the lower boundary. Furthermore, he was able to solve numerically for equilibrium of the (discretized) bus-corridor problem on the assumption that, at each bus stop, the same number of passengers board each bus picking up passengers at that stop, except perhaps the first. Accordingly, Arnott conjectured that the departure set takes the form shown in Figure 2, with the start of the rush hour endogenous and with no mass points on either boundary. We refer to this as the proposed departure set. Region I is the departure set, and Region II is the region in the $(0,0) \rightarrow(\bar{x}, \bar{t})$ rectangle below the departure set. The upper boundary of the departure set is the vehicle trajectory arriving at the CBD at $\bar{t}$, and includes departures from all locations. The departure set is connected; a vehicle trajectory (shown in Figure 2 as a dashed line) starts at $x=0$, in Region I is parallel to the upper boundary of the departure set (since, at each location, velocity is constant over that location's departure time interval), and in Region II accelerates. He conjectured that adjustment of the lower and upper boundaries provides enough freedom for the trip-timing condition to be satisfied for an arbitrary distribution of population along the corridor. For obvious reasons, Arnott termed the proposed departure set, a "horn-shaped" departure set.

As we shall see, Arnott's proposed departure set was incorrect in two respects. First, there must be a zone bordering the CBD with no departures. Second, even allowing for this, the modified departure set does not provide enough freedom for an equilibrium to exist with an arbitrary distribution of population along the corridor. Accordingly, we shall address the question: What distributions of population along the corridor are consistent with the modified departure set? Then, in the concluding remarks, we shall conjecture that the additional freedom needed to obtain equilibrium for other distributions of population can be obtained with mass points on the lower boundary of the departure set.

\section{Mathematical Analysis: Analytic Results}

In this section, we begin by analyzing the continuity equation within the two space-time regions, Region I (interior of the departure set) and Region II (exterior of the departure

\footnotetext{
${ }^{2}$ In the language of PDEs, the mass points along the lower boundary are sufficiently small that the characteristic line emanating from a point on the lower boundary (whose slope is completely determined by the density there) does not enter the departure set.
} 
set). We will show that the proposed departure set is not consistent with the TT condition, and we will present a modified departure set which is consistent. Furthermore, we will make the simplifying assumption that there are no mass points along the lower boundary of the departure set. Under this assumption, the entire traffic dynamics in both regions will be determined if we can specify both the population density and the lower boundary curve of the departure set. As we will see in (10), the width of the departure set and the population density uniquely determine the flow on the departure set, which includes its lower boundary under our assumption. Similarly, the width of the departure set and the specification of the flow on the lower boundary curve uniquely determine the population density. Thus, the entire traffic dynamics in Regions I and II and a unique population density will be determined if we can specify both the lower boundary curve of the departure set, and the flow along this lower boundary curve. Their specification must be consistent with the continuity equations in both regions, and also with the TT condition.

At the conclusion of this section we will present three equations, (12), (13) and (14), which must be satisfied by a lower boundary curve of a modified departure set and a flow along the lower boundary curve. What will not be clear from our analytic results, is whether or not these three equations admit a solution, and, if so, whether or not they admit a unique solution.

\subsection{Continuity Equation: Method of Characteristics}

The continuity equations in Regions I and II are first-order, quasi-linear partial differential equations for the density, $\rho$, as a function of $(x, t)$ within each region. We may solve each equation by the method of characteristics, which converts the PDE into a system of ODE's, whose solution yields characteristic curves in the $x$ - $t$ plane, with $\rho$ determined in each region as a function along these curves (Evans (2002), Rhee, Aris and Amundson (1986)). In the following two sections we apply this method of characteristics to Regions I and II.

\subsection{Region I}

The TT condition implies that the departure rate is independent of time over the interior and on the upper boundary of the departure set. Since we further assume that the departure rate is independent of time on the lower boundary of the departure set, the continuity equation in Region I is

$$
\frac{\partial \rho}{\partial t}+(\rho V)^{\prime} \frac{\partial \rho}{\partial x}=n(x)
$$

where / indicates a derivative with respect to $\rho$, i.e., $(\rho V)^{\prime} \equiv \frac{d}{d \rho}(\rho V)$. The characteristic curves for this PDE satisfy

$$
\frac{d t}{1}=\frac{d x}{(\rho V)^{\prime}}=\frac{d \rho}{n(x)}
$$

In particular, the flow, $F=\rho V$, satisfies

$$
(\rho V)^{\prime} d \rho=n(x) d x
$$


and, since initially $F=0$,

$$
F(x)=(\rho V)(x)=\int_{0}^{x} n\left(x^{\prime}\right) d x^{\prime}
$$

Thus, flow on the departure set is uniquely determined by the departure rate. By differentiating this equation we see that the departure rate is also uniquely determined from the flow. Since we have already established that, over the departure set, the departure rate is constant at each location, if the width of the departure set is known then the flow in Region I and the population density are equivalent, i.e., knowledge of one uniquely determines the other.

In addition, since the population is nonzero at all locations, (10) implies that within the departure set, flow is a strictly increasing function of $x$. Since we are assuming classical flow technology such that velocity decreases with density and becomes zero at jam density, the flow-density and flow-velocity curves will generally have the shapes similar to those shown in Figure 3. Since flow is increasing in the departure set, there is a maximum density (and corresponding minimum velocity) which may be achieved within the departure set. This point corresponds to the border point between congested traffic and hypercongested traffic, and is called capacity flow. Hence, we conclude that an equilibrium solution to the
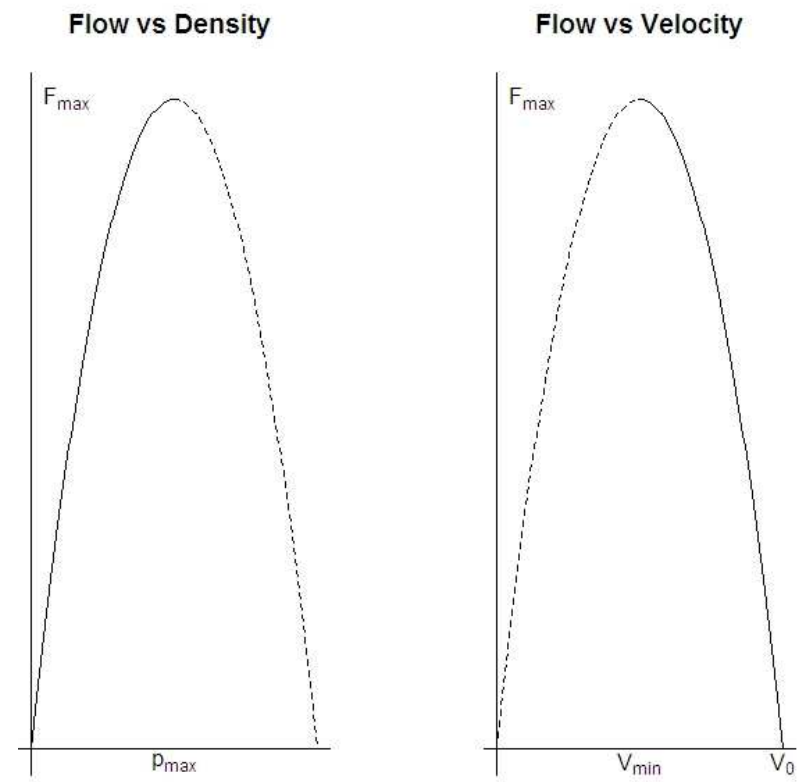

Figure 3: Congestion (solid line) and hypercongestion (dashed line). Peak of the graphs corresponds to the border point between congestion and hypercongestion, i.e., the point of capacity flow. Since flow is strictly increasing within the departure set, hypercongestion will not occur.

Corridor Problem does not permit hypercongestion. Furthermore, this implies a one-to-one relationship within the departure set between flow, velocity and density. 


\subsection{Region II}

The continuity equation in Region II is the well-known homogeneous equation

$$
\frac{\partial \rho}{\partial t}+(\rho V)^{\prime} \frac{\partial \rho}{\partial x}=0
$$

$(\rho V)^{\prime}$ is the rate at which flow changes with density. Thus, its reciprocal is the rate at which density changes with flow. As discussed in Newell (1993), the characteristic curves in Region II are iso-density curves which are straight lines with $t$ - $x$ slope, $\frac{d t}{d x}=\frac{1}{(\rho V)^{\prime}}$. These characteristic lines emanate from the lower boundary of the departure set, and completely determine the density field in Region II (see Figure 4). Since flow is non-decreasing on the

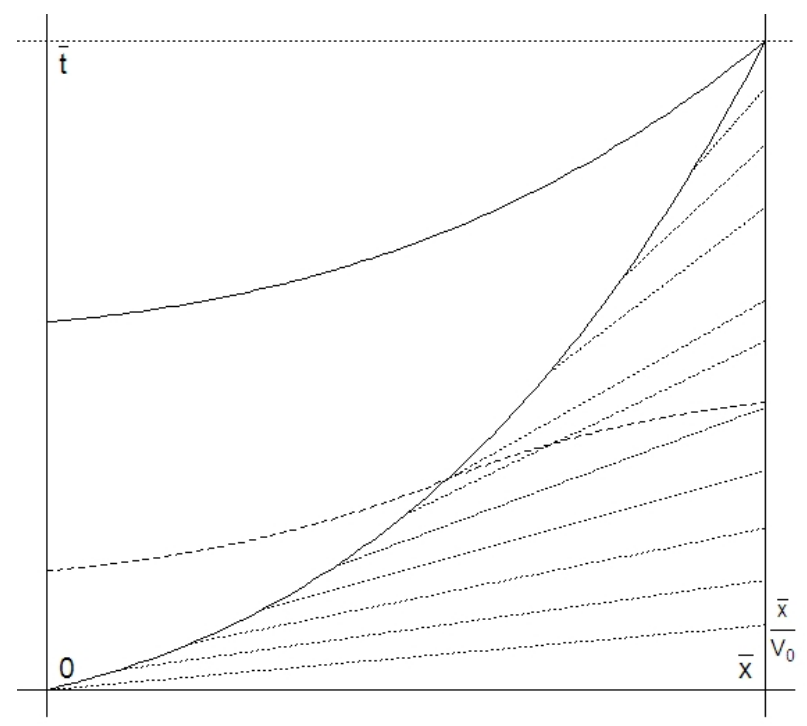

Figure 4: Characteristic lines in Region II, which are iso-density curves, along with a sample vehicle trajectory.

lower boundary of the departure set, the slopes of the characteristic lines are non-decreasing, and therefore the characteristic lines are non-intersecting. This excludes the possibility of shocks occurring in Region II, and implies that density is continuous in Region II. Note that the initial vehicle trajectory, with $V=V_{0}$, coincides with a characteristic line. Also note that the slope of a characteristic line at a point on the lower boundary is greater than the slope of a trajectory curve at that point, since $\frac{1}{(\rho V)^{\prime}}=\frac{1}{V+\rho V^{\prime}}>\frac{1}{V}$.

\subsection{Parametrization of Lower Boundary Curve}

We parametrize the lower boundary curve of the departure set as follows (refer to Figure 5). A cohort of vehicles departs $x=0$ at time $u$, reaches the lower boundary curve of the departure set at location $x=b(u)$, and arrives at the CBD at time $a$. A consequence of the TT condition, (4), is $a=\frac{\alpha}{\alpha-\beta} u+\frac{\bar{x}}{V_{0}}$. Note that we have normalized time so that the start of the rush hour is $t=0$, and that the total length of the rush hour, $\bar{t}$, will be determined as 
part of the overall solution. The travel time to the lower boundary curve is

$$
T_{I}(u)=\int_{0}^{b(u)} \frac{1}{v\left(x^{\prime}\right)} d x^{\prime}
$$

where $v(x)$ is the velocity in the departure set at location $x$. Thus, the $(x, t)$ coordinates along the lower boundary curve are parametrized as $\left(b(u), u+T_{I}(u)\right)$.

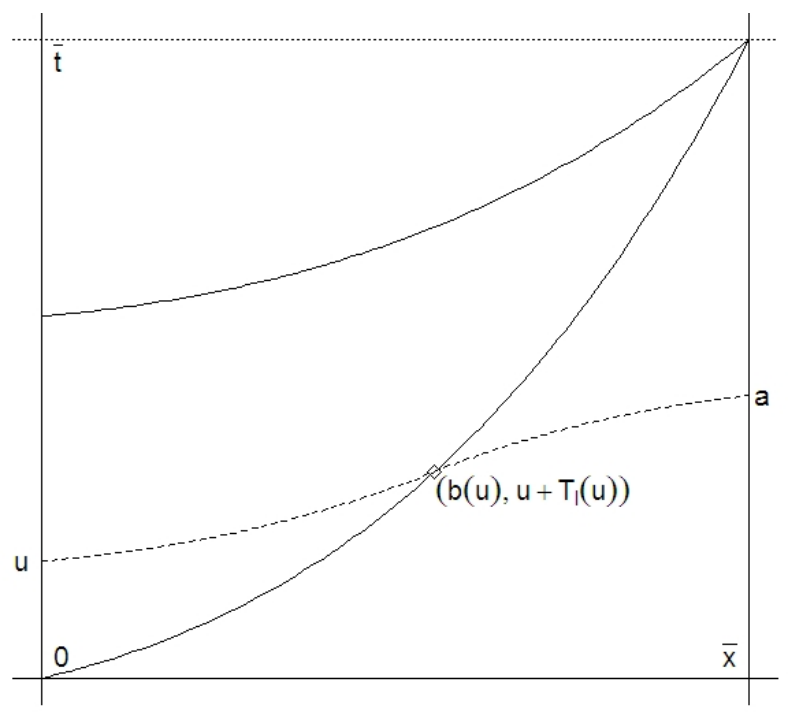

Figure 5: Trajectory departing $x=0$ reaches the lower boundary at location $x=b(u)$. Velocity distribution within departure set at location $x$ is $v(x)=v(b(u))$.

\subsection{Arrival Flow Rate}

An important consequence of the TT condition is that it enables us to determine the flow rate at the CBD in terms of the flow rate at the lower boundary of the departure set. Let $F(b(u))$ denote the flow within the departure set at location $x=b(u)$, so $F(b(u))=\int_{0}^{b(u)} n\left(x^{\prime}\right) d x^{\prime}$. If we follow the vehicle trajectory which departs $x=0$ at time $u$, leaves the departure set at location $b(u)$, and arrives at the CBD at time $a(u)=\frac{\alpha}{\alpha-\beta} u+\frac{\bar{x}}{V_{0}}$, then we may track the cumulative number of arrivals to the CBD by time $a, A(a)$,

$$
\begin{aligned}
A(a) & =\int_{0}^{u(a)} \int_{0}^{b\left(u^{\prime}\right)} n(x) d x d u^{\prime} \\
& =\int_{0}^{u(a)} F\left(b\left(u^{\prime}\right)\right) d u^{\prime} .
\end{aligned}
$$

Since $\frac{d u}{d a}=\frac{\alpha-\beta}{\alpha}$, we may determine the arrival flow rate at the CBD as

$$
\text { Flow at } \begin{aligned}
(\bar{x}, a) & =\frac{d A}{d a}=F(b(u(a))) \frac{d u}{d a} \\
& =\frac{\alpha-\beta}{\alpha} F(b(u(a)))
\end{aligned}
$$


Hence, flow for a cohort of vehicles strictly decreases from the lower boundary to the CBD by the multiplicative factor $\frac{\alpha-\beta}{\alpha}$.

\subsection{Modification of Proposed Departure Set}

The flow of a cohort of vehicles decreases from the lower boundary of the departure set to the CBD by a factor of $\frac{\alpha-\beta}{\alpha}$. Also, under the assumption that there are no mass points along the lower boundary of the departure set, flow must be continuous from the lower boundary of the departure set to the CBD. Since these two properties cannot be simultaneously satisfied for the last cohort of vehicles, the proposed, horn-shaped departure set is inconsistent with equilibrium. If the departure set is modified so that there is zero population density over an interval before the CBD, with the interval being determined so as to satisfy the first condition, then both conditions can be satisfied. Thus, in what follows we consider a modified departure set, which is still horn-shaped and which still has no mass points on its lower boundary, but has zero population density near the CBD. A modified departure set is shown in Figure 6 .

Since flow is an increasing function along the lower boundary curve and since hypercongestion does not occur, the maximum flow must occur at the tip of the horn, and this maximum flow must be less than or equal to capacity flow.

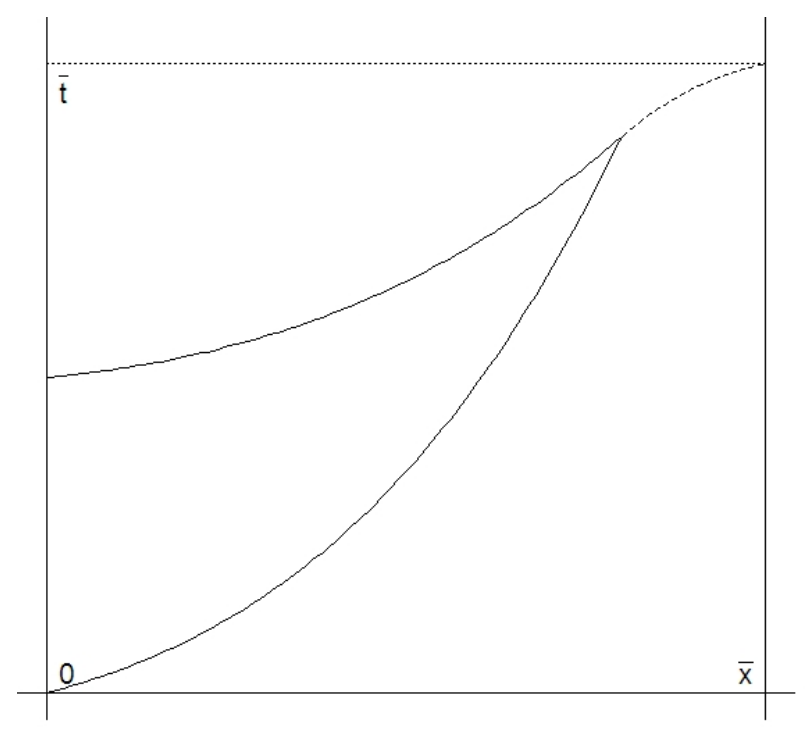

Figure 6: The flow for a cohort must decrease from the tip of the departure set to the CBD by the multiplicative factor $\frac{\alpha-\beta}{\alpha}$.

\subsection{Three Governing Equations: Summary}

The TT condition implies that the departure rate at each location is constant over the interior of the departure set and along the upper boundary of the departure set. Since we are extending this implication to the lower boundary of the departure set, i.e., since we are excluding the possibility of mass points along the lower boundary, we can derive three 
governing equations, (12), (13) and (14), that must be satisfied by an equilibrium solution to the Corridor Problem with the modified departure set.

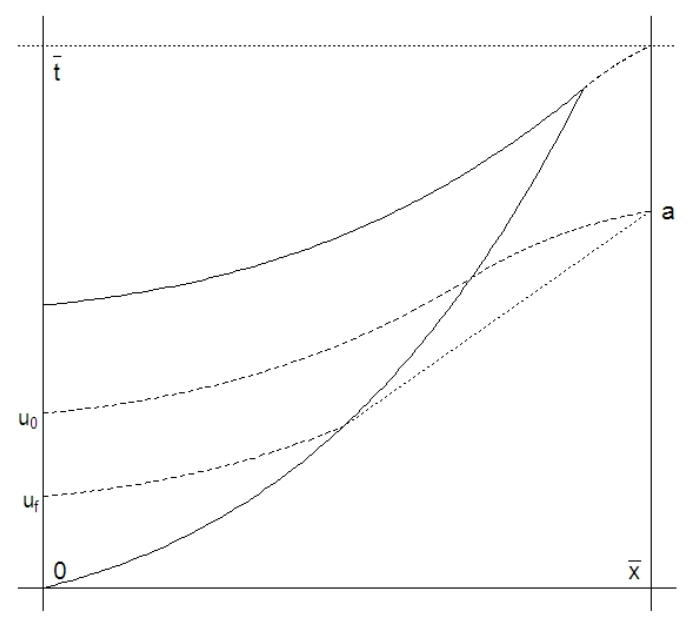

Figure 7: Arrival of the vehicle trajectory departing $x=0$ at time $u_{0}$ intersects the characteristic line which originates from $\left(b\left(u_{f}\right), u_{f}+T_{I}\left(u_{f}\right)\right)$, where $u_{f}<u_{0}$.

Consider a vehicle trajectory departing $x=0$ at time $u_{0}$ and arriving at the CBD at time $a$ (Figure 7). The characteristic curve through this arrival point originates from the lower boundary at location $b\left(u_{f}\right)$, where $u_{f}<u_{0}$ (see Figure 7 ). Since the characteristic curve is a straight line of constant flow, we may equate the flow at the lower boundary for the cohort $u_{f}$ to the flow at the CBD for the cohort $u_{0}$. Hence, for each departure time $u_{0}$ there will correspond a unique value of $u_{f}$. We now state the three governing equations which must be satisfied for each pair of $u_{0}$ and $u_{f}$ values, and we derive these equations in the following sections.

$$
\begin{gathered}
u_{0}=\frac{\alpha-\beta}{\alpha}\left[\frac{\bar{x}-b\left(u_{f}\right)}{\left.\frac{d}{d \rho}(\rho V)\right|_{b\left(u_{f}\right)}}+u_{f}+\int_{0}^{b\left(u_{f}\right)} \frac{1}{v\left(x^{\prime}\right)} d x^{\prime}-\frac{\bar{x}}{V_{0}}\right] \\
F\left(b\left(u_{f}\right)\right)=\frac{\alpha-\beta}{\alpha} F\left(b\left(u_{0}\right)\right) \\
\int_{u_{f}}^{u_{0}} F\left(b\left(u^{\prime}\right)\right) d u^{\prime}=\left.\left(-\rho+\frac{\rho V}{(\rho V)^{\prime}}\right)\right|_{b\left(u_{f}\right)}\left(\bar{x}-b\left(u_{f}\right)\right) .
\end{gathered}
$$

Since we have established a one-to-one correspondence within the departure set between density, flow and velocity, we can eliminate all the terms involving density, $\rho$, and velocity, 
$V$, and rewrite all three equations in terms of only flow, $F$. A solution to these three equations consists of the function $b(u)$ describing the $x$-coordinates of the lower boundary curve, and the flow function along the lower boundary curve, $F(b(u))$. Since the outer boundary of residential settlement is at $x=0$, the function $b(u)$ must satisfy $b(0)=0$, and since initially there is no traffic, the flow function must satisfy $F(0)=0$. Note that the initial width of the departure set and the work start-time at the CBD, $\bar{t}$, will be determined as part of the solution and are not given a priori.

To summarize, the Corridor Problem with the modified departure set, which excludes the possibility of mass points along the lower boundary, divides the space-time plane into two regions. In Region I traffic must satisfy an inhomogeneous continuity equation, and in Region II the homogeneous continuity equation. The equality component of the TT is a global condition imposed on the vehicle trajectories. We have reduced the problem to solving for the lower boundary curve of the departure set, $b(u)$, and a flow along this lower boundary curve, $F(b(u))$, subject to the constraints $b(0)=0$ and $F(0)=0$. The solution of these two quantities must simultaneously satisfy three nonlinear equations, (12), (13) and (14), and, furthermore, their solution will uniquely specify a population density.

\subsection{Derivation of the Three Governing Equations}

\section{First Governing Equation}

Referring back to Figure 7, since the TT condition implies that $a=\frac{\alpha}{\alpha-\beta} u_{0}+\frac{\bar{x}}{V_{0}}$, and since the slope of the characteristic line is $\frac{1}{\left.\frac{d}{d \rho}(\rho V)\right|_{b\left(u_{f}\right)}}$, by calculating the slope directly we have

$$
\frac{\frac{\alpha}{\alpha-\beta} u_{0}+\frac{\bar{x}}{V_{0}}-\left[u_{f}+T_{I}\left(u_{f}\right)\right]}{\bar{x}-b\left(u_{f}\right)}=\frac{1}{\left.\frac{d}{d \rho}(\rho V)\right|_{b\left(u_{f}\right)}} .
$$

We may solve for $u_{0}$ in terms of $u_{f}$ to obtain the first governing equation

$$
u_{0}=\frac{\alpha-\beta}{\alpha}\left[\frac{\bar{x}-b\left(u_{f}\right)}{\left.\frac{d}{d \rho}(\rho V)\right|_{b\left(u_{f}\right)}}+u_{f}+\int_{0}^{b\left(u_{f}\right)} \frac{1}{v\left(x^{\prime}\right)} d x^{\prime}-\frac{\bar{x}}{V_{0}}\right] .
$$

\section{Second Governing Equation}

Flow on the lower boundary decreases along a trajectory to the CBD by the multiplicative factor $\frac{\alpha-\beta}{\alpha}$, i.e., if $F(b(u))$ is the flow at the lower boundary of the cohort that departed at time $u$, and $a(u)=\frac{\alpha}{\alpha-\beta} u+\frac{\bar{x}}{V_{0}}$ is the arrival time at the CBD of this cohort, then the flow at $(\bar{x}, a(u))$ equals $\frac{\alpha-\beta}{\alpha} F(b(u))$. Referring to Figure 7 , since characteristic lines in Region II are iso-flow lines, this allows us to express the flow within the departure set at location $b\left(u_{f}\right)$ in terms of the flow within the departure set at location $b\left(u_{0}\right)$, yielding the second governing equation

$$
F\left(b\left(u_{f}\right)\right)=\frac{\alpha-\beta}{\alpha} F\left(b\left(u_{0}\right)\right) .
$$

Since hypercongestion does not occur within the departure set, there is a one-to-one relation between flow, density and velocity. We may therefore also use this second governing equation to determine the density (or velocity) at $b\left(u_{f}\right)$ in terms of the density (or velocity) at $b\left(u_{0}\right)$. 


\section{Third Governing Equation}

Consider a trajectory which departs $x=0$ at time $u_{0}$, and parameterize the $(x, t)$ coordinates of the portion of this trajectory in Region II as $(\tilde{x}(u), \tilde{t}(u)), u_{f} \leq u \leq u_{0}$, where $(\tilde{x}(u), \tilde{t}(u))$ is the point on the trajectory in Region II which intersects the characteristic line emanating from the lower boundary of the departure set at the point $\left(b(u), u+T_{I}(u)\right)$ (see Figure 8).

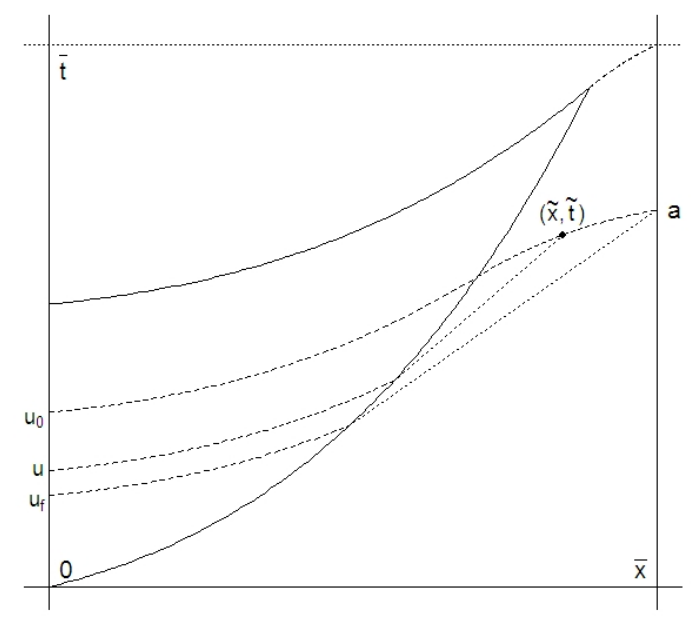

Figure 8: Trajectory in Region II parametrized as $(\tilde{x}(u), \tilde{t}(u))$, where $u_{f} \leq u \leq u_{0}$.

Hence,

$$
\begin{aligned}
& \left(\tilde{x}\left(u_{0}\right), \tilde{t}\left(u_{0}\right)\right)=\left(b\left(u_{0}\right), u_{0}+T_{I}\left(u_{0}\right)\right) \\
& \left(\tilde{x}\left(u_{f}\right), \tilde{t}\left(u_{f}\right)\right)=\left(\bar{x}, \frac{\alpha}{\alpha-\beta} u_{0}+\frac{\bar{x}}{V_{0}}\right) .
\end{aligned}
$$

The cumulative flow, or cumulative number of arrivals, is constant along a trajectory in Region II. By (11), for the cohort which departs $x=0$ at time $u$ the cumulative flow along the cohort's trajectory in Region II is

$$
A(u)=\int_{0}^{u} F\left(b\left(u^{\prime}\right)\right) d u^{\prime} .
$$

Denote the cumulative flow as a function of the space-time coordinate in Region II as $\hat{A}(x, t)$, to distinguish it from the cumulative flow along a trajectory in Region II, $A(u)$. Following Newell (1993), if we move along a characteristic line in Region II from $(x, t)$ to $(x+d x, t+d t)$, then the cumulative flow along this line satisfies

$$
\begin{gathered}
\frac{d \hat{A}}{d x}=-\rho+\frac{\rho V}{(\rho V)^{\prime}} \\
\frac{d \hat{A}}{d t}=-\rho(\rho V)^{\prime}+\rho V,
\end{gathered}
$$


where $\rho$ and $V$ are the constant density and constant velocity along the characteristic line. If we integrate (15a) along the characteristic line from $\left(b(u), u+T_{I}(u)\right)$ to $(\tilde{x}(u), \tilde{t}(u))$, we obtain

$$
\hat{A}(\tilde{x}(u), \tilde{t}(u))-\hat{A}\left(b(u), u+T_{I}(u)\right)=\left.\left(-\rho+\frac{\rho V}{(\rho V)^{\prime}}\right)\right|_{b(u)}(\tilde{x}(u)-b(u)), \quad u_{f} \leq u \leq u_{0} .
$$

Since along the trajectory $(\tilde{x}(u), \tilde{t}(u))$ the cumulative flow is the constant value $A\left(u_{0}\right)=$ $\int_{0}^{u_{0}} F\left(b\left(u^{\prime}\right)\right) d u^{\prime}$, and since on the lower boundary at $b(u)$ the cumulative flow is $A(u)=$ $\int_{0}^{u} F\left(b\left(u^{\prime}\right)\right) d u^{\prime}$, we may rewrite this expression as

$$
\int_{u}^{u_{0}} F\left(b\left(u^{\prime}\right)\right) d u^{\prime}=\left.\left(-\rho+\frac{\rho V}{(\rho V)^{\prime}}\right)\right|_{b(u)}(\tilde{x}(u)-b(u)), \quad u_{f} \leq u \leq u_{0} .
$$

In particular, when $u=u_{f}$, we obtain the third governing equation

$$
\int_{u_{f}}^{u_{0}} F\left(b\left(u^{\prime}\right)\right) d u^{\prime}=\left.\left(-\rho+\frac{\rho V}{(\rho V)^{\prime}}\right)\right|_{b\left(u_{f}\right)}\left(\bar{x}-b\left(u_{f}\right)\right) .
$$

This equation relates the integral of the flow along the lower boundary from $b\left(u_{f}\right)$ to $b\left(u_{0}\right)$, to the distance from the CBD to the lower boundary at $b\left(u_{f}\right)$, and the density and velocity at $b\left(u_{f}\right)$.

\section{Numerical Analysis (Greenshields')}

Ideally, given an arbitrary population density we would like to construct a solution to the Corridor Problem and, if multiple solutions exist, characterize all possible solutions. However, it is not clear if a solution will exist for an arbitrary population density, e.g., we have already shown that the population must be zero some finite distance before the CBD. In the last section we showed how a solution with the modified departure set can be determined by solving for a lower boundary curve of the departure set and a flow along this lower boundary curve. We also showed how a specific solution uniquely determines a population density. Thus, we seek to characterize all possible solutions consisting of lower boundary curves and flows along these lower boundary curves, from which we could extract all possible population densities which admit solutions to the Corridor Problem with the modified departure set. We have determined that any solution must satisfy the three governing equations. What is not clear, however, is whether or not the three governing equations admit a solution, and, if so, whether or not they admit a unique solution?

In this section we consider a specific velocity-density relation (Greenshields'), and provide a numerically constructive proof that, for a given ratio of parameters, $\frac{\beta}{\alpha}$, the three governing equations admit a unique solution for a modified departure set and a flow distribution on that departure set that reaches capacity flow (note that $\bar{x}$ and $V_{0}$ are scale parameters that will only determine the scaling along the distance and time axes). As discussed, this solution will uniquely determine a population density. Thus, in this section we will conclude that, using Greenshields' relation and given $\frac{\beta}{\alpha}$, there is a unique population density which admits 
a solution to the Corridor Problem with the modified departure set that reaches capacity flow.

Each one of these solutions admits a continuous family of truncated solutions which do not reach capacity flow. To see this, suppose that we have a departure set solution which reaches capacity flow (which must necessarily occur at the tip of the departure set, since flow is non-decreasing). Consider any vehicle trajectory which departs $x=0$ before the last departure, i.e., below the upper boundary of the departure set. This vehicle trajectory intersects the lower boundary of the departure set at some midway point, and traverses Region II to reach the CBD. If we now let this vehicle trajectory be the upper boundary of a new, truncated departure set, removing all other trajectories which depart after it, then we obtain a truncated departure set solution which is identical to the original departure set over the regions not truncated. The flow at the tip of this truncated departure set is less than capacity flow. Based on the results of this section, we can further conclude (with Greenshields' relation) that, for a given flow value less than or equal to capacity flow at the tip of the horn and a given $\frac{\beta}{\alpha}$, there is a unique population distribution solving the Corridor Problem with the modified departure set.

We begin by introducing Greenshields' relation, choosing appropriate scale parameters, and then restating the three governing equations with these relations and scale parameters implemented. We then give a broad overview of our numerical strategy before presenting the details.

\subsection{Greenshields' Velocity-Density Relation}

Greenshields' linear velocity-density relation is

$$
\begin{aligned}
& V=V_{0}\left(1-\frac{\rho}{\rho_{J}}\right) \\
& \rho=\rho_{J}\left(1-\frac{V}{V_{0}}\right),
\end{aligned}
$$

where $V_{0}$ is the free-flow velocity and $\rho_{J}$ is the jam density. We write the flow in terms of velocity as

$$
F=\rho V=\frac{\rho_{J}}{V_{0}} V\left(V_{0}-V\right)
$$

which achieves its maximum value, capacity flow, at $V=\frac{V_{0}}{2}$, Since we have shown that hypercongestion does not occur, $\frac{V_{0}}{2} \leq V \leq V_{0}$, and we may write velocity in terms of flow as

$$
V=\frac{V_{0}}{2}\left(1+\sqrt{1-\frac{4 F}{\rho_{J} V_{0}}}\right) .
$$

Also,

$$
(\rho V)^{\prime} \equiv \frac{d}{d \rho}(\rho V)=2 V-V_{0}
$$




\subsection{Scale Parameters and Notation}

The natural units of distance and time are $\bar{x}$ and $\frac{\bar{x}}{V_{0}}$, respectively. Thus, we choose our units such that $\bar{x}=1$ and $\frac{\bar{x}}{V_{0}}=1$, so that $V_{0}=1$ and $\frac{1}{2} \leq V \leq 1$. We also choose the units of population so that the jam density, $\rho_{J}=4$, which results in the flow, $F$ varying from 0 to a capacity flow value of 1 . The only relevant parameter, then, is $\frac{\beta}{\alpha}<1$, which is the ratio of the unit time early cost to the unit travel time cost. Finally, let $w$ denote the slope of the flow vs. density curve, $w=2 V-1$ where $0 \leq w \leq 1$. Newell (1993) refers to $w$ as the "wave velocity," and although it is not necessary to introduce this additional function, it is useful in simplifying the algebraic manipulations that follow. Using these units and notation we restate the three governing equations in a form that will be useful for our numerical procedure:

$$
\begin{gathered}
u_{0}=\frac{\alpha-\beta}{\alpha}\left[\frac{1-b\left(u_{f}\right)}{w\left(b\left(u_{f}\right)\right)}+u_{f}+\int_{0}^{b\left(u_{f}\right)} \frac{2}{1+\sqrt{1-F\left(x^{\prime}\right)}} d x^{\prime}-1\right] \\
F\left(b\left(u_{f}\right)\right)=\frac{\alpha-\beta}{\alpha} F\left(b\left(u_{0}\right)\right) \\
\int_{u_{f}}^{u_{0}} F\left(b\left(u^{\prime}\right)\right) d u^{\prime}=\frac{\left(1-w\left(b\left(u_{f}\right)\right)\right)^{2}}{w\left(b\left(u_{f}\right)\right)}\left(1-b\left(u_{f}\right)\right) .
\end{gathered}
$$

\subsection{Overview of Numerical Solution}

To determine any solutions to the Corridor Problem with the modified departure set under Greenshields' relation, we must simultaneously solve (17) subject to the constraints $b(0)=0$ and $F(0)=0$. We will first seek a departure set solution which reaches capacity flow. As mentioned earlier, the existence of such a solution will imply a continuous family of truncated solutions which do not reach capacity flow.

(17) involve a natural pairing of $u$ values, $u_{0}$ and $u_{f}$. We utilize this pairing to discretize the problem, using the second governing equation (17b) to exactly determine the flow values at each of the discretization points, with flow values ranging from 0 to the capacity flow value of 1 . If our discretization is fine enough, then we can linearly approximate both the lower boundary curve and the flow over each discretized subinterval, which enables us to "discretize" the first and third governing equations, (17a) and (17c), i.e., to restate them in a form on each discretized subinterval which does not involve integrals. Our numerical procedure takes the lower boundary curve and flow values at one discretized subinterval, inputs them into the discretized versions of the first and third governing equations for the next discretized subinterval, yielding a pair of linear equations for the lower boundary curve and flow values for the next discretized subinterval. The unique solution to this pair of linear equations yields the lower boundary curve and flow values for the next discretized subinterval. Futhermore, the initial seed values for this numerical procedure are uniquely determined by linearly approximating the lower boundary curve and flow values on the first discretized subinterval. Hence, by making valid linear approximations we numerically construct a solution to the three governing equations, and at each step of our numerical procedure the solution values we obtain are uniquely determined. Thus, this procedure provides a numerically constructive proof that, given Greenshields' relation and a ratio of 
parameters $\frac{\beta}{\alpha}$, there is a unique solution to the Corridor Problem with the modified departure set that reaches capacity flow.

A complication arises when we attempt to construct the final segment of the lower boundary curve, since the $u_{f}$ values in this segment do not have a corresponding $u_{0}$ value with which they can be paired, and thus the first and third governing equations will no longer be applicable. We alleviate this problem by guessing a value of the lower boundary curve for the subsequent discretized subinterval, using our guess to numerically construct a vehicle trajectory which should theoretically intersect the lower boundary curve exactly at the point which we guessed, and choosing repeated guesses until we find that the vehicle trajectory does intersect our point at some desired level of tolerance. This procedure is re-iterated until the final segment has been constructed. The following sections provide the details of the numerical procedure.

\subsection{Iterated Sequence of Discretized Flow Values}

Over the departure set, we seek a solution such that the flow increases from 0 to a capacity flow value of 1 . We choose an initial flow value, $0<F_{0} \leq 1$, determine the point on the lower boundary curve of the departure set where this flow value is attained, track the trajectory curve through this point until it reaches the CBD, and then backtrack along the characteristic line intersecting this point until reaching the lower boundary curve. This has already been graphically illustrated in Figure 7. By $(17 \mathrm{~b})$, the flow at this new iterated value is $F_{1}=F_{0}\left(\frac{\alpha-\beta}{\alpha}\right)$. Continuing this iterative procedure, as we approach the residential boundary $(x=0)$, the nth iterated flow value is $F_{n}=F_{0}\left(\frac{\alpha-\beta}{\alpha}\right)^{n}$, which approaches 0 .

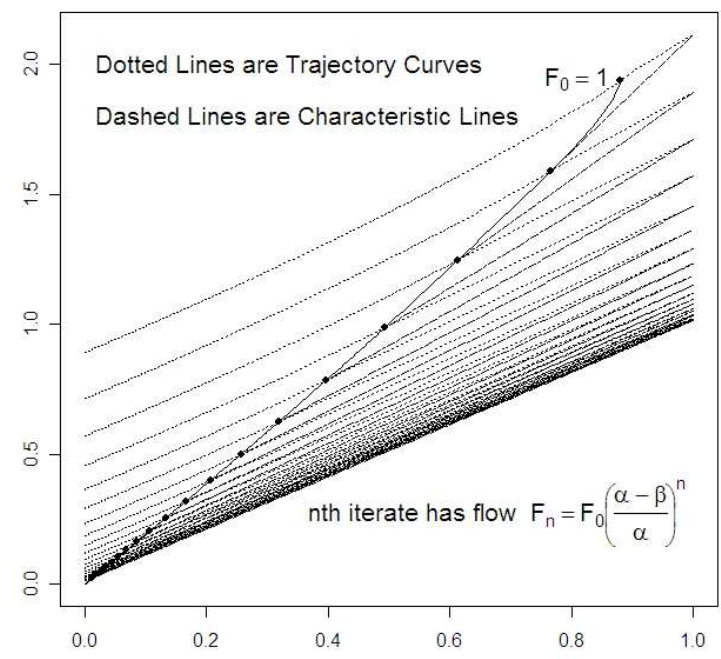

Figure 9: Iterated flow values. Iterative procedure tracks the intersection at the CBD of a trajectory curve with a characteristic line.

We graphically illustrate this iterative procedure in Figure 9, with an initial flow value of 
$F_{0}=1$, capacity flow. Note that $(17 \mathrm{~b})$ allows us to develop a sequence of flow iterates, $F_{i} \equiv F\left(b\left(u_{i}\right)\right)$, without knowing the corresponding $u_{i}$ and $b_{i} \equiv b\left(u_{i}\right)$ values.

As illustrated in Figure 9, if we begin with $F_{0}=1$ and iterate this procedure $N$ times, then we partition the lower boundary curve into $N+1$ segments, where the first segment begins with a flow value of 1 and ends with a flow value of $\frac{\alpha-\beta}{\alpha}$. We further subdivide the flow values on this first segment into $k$ subdivisions, equally spaced between the values $\frac{\alpha-\beta}{\alpha}$ and 1 . We apply the iterative procedure to each of the $k$ flow values within this segment, generating $k$ flow values within each of the subsequent segments. Hence, if we consider the first $N$ segments constructed, each having $k$ subdivisions, then we obtain a sequence with a total of $N_{f}=N k$ flow values. We illustrate this idea in Figure 10, where we have reordered the sequence of flow values such that $F_{1}$ is the smallest flow value in the sequence, increasing to the capacity flow value, $F_{N_{f}}=F_{N k}=1$. The generation of this sequence of flow iterates

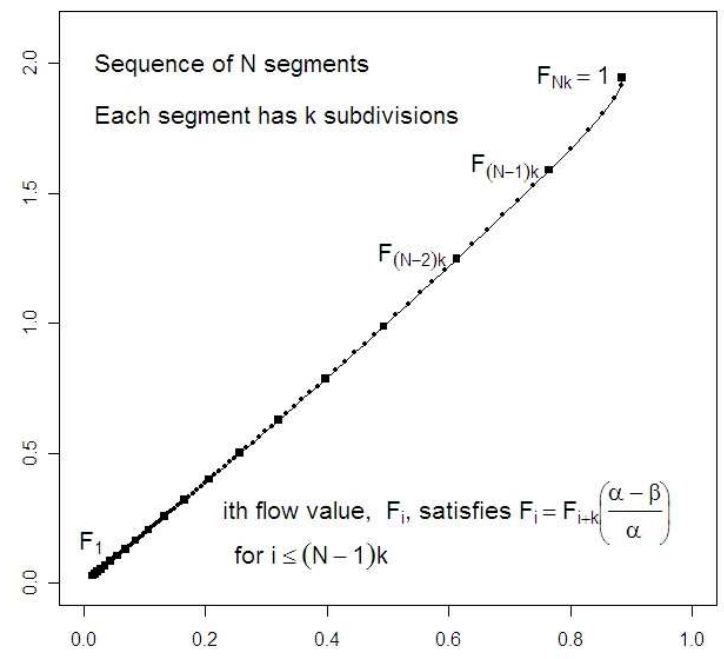

Figure 10: Sequence of flow values from $F_{1}$ to $F_{N_{f}}=F_{N k}=1$. For all $i, 1 \leq i \leq(N-1) k$, the $i$ th flow value satisfies $F_{i}=F_{i+k}\left(\frac{\alpha-\beta}{\alpha}\right)$.

derives from (17b), and does not require knowledge of the corresponding $u_{i}$ or $b\left(u_{i}\right)$ values. We state a closed form formula for all flow values in this sequence, given values of $N$ and $k$ :

$$
F_{i+(N-j) k}=\left(\frac{\alpha-\beta}{\alpha}\right)^{j-1}\left(\frac{\alpha-\beta}{\alpha}+\frac{i}{k}\left[1-\frac{\alpha-\beta}{\alpha}\right]\right), \quad \forall 1 \leq j \leq N, 1 \leq i \leq k
$$

Since $V=\frac{1}{2}(1+\sqrt{1-F})$ and $w=\sqrt{1-F}$, we may use this sequence to obtain corresponding sequences of $v_{i} \equiv v\left(b\left(u_{i}\right)\right)$ and $w_{i} \equiv w\left(b\left(u_{i}\right)\right)$ values. 


\subsection{Discretization of the Third Governing Equation}

Let $A_{i} \equiv A\left(u_{i}\right)=\int_{0}^{u_{i}} F\left(b\left(u^{\prime}\right)\right) d u^{\prime}$. From the third governing equation, (17c), for all $i$, $1 \leq i \leq N_{f}-k$

$$
\int_{u_{i}}^{u_{i+k}} F\left(b\left(u^{\prime}\right)\right) d u^{\prime}=A_{i+k}-A_{i}=\frac{\left(1-w_{i}\right)^{2}}{w_{i}}\left(1-b_{i}\right) .
$$

If we choose $k$ large enough, then over each subinterval $\left(u_{i-1}, u_{i}\right)$, we may approximate $F(b(u))$ as a linear function of $u$,

$$
F(b(u)) \approx F_{i-1}+\frac{F_{i}-F_{i-1}}{u_{i}-u_{i-1}}\left(u-u_{i-1}\right), \quad u \in\left(u_{i-1}, u_{i}\right) .
$$

We use this linear approximation to directly approximate $A_{i+k}$ as

$$
\begin{aligned}
A_{i+k} & =\int_{0}^{u_{i+k}} F\left(b\left(u^{\prime}\right)\right) d u^{\prime} \\
& =A_{i+k-1}+\int_{u_{i+k-1}}^{u_{i+k}} F\left(b\left(u^{\prime}\right)\right) d u^{\prime} \\
& \approx A_{i+k-1}+\frac{u_{i+k}-u_{i+k-1}}{2}\left(F_{i+k}+F_{i+k-1}\right) .
\end{aligned}
$$

Substituting this expression for $A_{i+k}$ into (19) yields

$$
A_{i+k-1}+\frac{u_{i+k}-u_{i+k-1}}{2}\left(F_{i+k}+F_{i+k-1}\right)-A_{i}=\frac{\left(1-w_{i}\right)^{2}}{w_{i}}\left(1-b_{i}\right) .
$$

We rearrange this equation to solve for $u_{i+k}$, yielding our discretized version of the third governing equation:

$$
u_{i+k}=u_{i+k-1}+\frac{2}{F_{i+k}+F_{i+k-1}}\left[\frac{\left(1-w_{i}\right)^{2}}{w_{i}}\left(1-b_{i}\right)-A_{i+k-1}+A_{i}\right], \quad \forall i, 1 \leq i \leq N f-k .
$$

\subsection{Discretization of the First Governing Equation}

The $(x, t)$ coordinates of the lower boundary curve are parametrized in terms of $u$ as $(b(u), u+$

$\left.T_{I}(u)\right)=\left(b(u), u+\int_{0}^{b(u)} \frac{d x^{\prime}}{v\left(x^{\prime}\right)}\right)$. Corresponding to our sequence of flow values, we denote the sequence of coordinates of the lower boundary curve as $\left(b_{i}, t_{i}\right), 1 \leq i \leq N_{f}$. The time coordinates, $t_{i}$, can be expressed as

$$
\begin{aligned}
t_{i} & =u_{i}+\int_{0}^{b_{i}} \frac{2}{1+\sqrt{1-F\left(x^{\prime}\right)}} d x^{\prime} \\
& =u_{i-1}+\int_{0}^{b_{i-1}} \frac{2}{1+\sqrt{1-F\left(x^{\prime}\right)}} d x^{\prime}+\left(u_{i}-u_{i-1}\right)+\int_{b_{i-1}}^{b_{i}} \frac{2}{1+\sqrt{1-F\left(x^{\prime}\right)}} d x^{\prime} \\
& =t_{i-1}+\left(u_{i}-u_{i-1}\right)+\int_{b_{i-1}}^{b_{i}} \frac{2}{1+\sqrt{1-F\left(x^{\prime}\right)}} d x^{\prime} .
\end{aligned}
$$


If we choose $k$ large enough, then over each subinterval $\left(u_{i-1}, u_{i}\right)$, we may approximate $F(b(u))$ and $b(u)$ as linear functions of $u$ :

$$
\begin{gathered}
F(b(u)) \approx F_{i-1}+\frac{F_{i}-F_{i-1}}{u_{i}-u_{i-1}}\left(u-u_{i-1}\right), \quad u \in\left(u_{i-1}, u_{i}\right) \\
b(u) \approx b_{i-1}+\frac{b_{i}-b_{i-1}}{u_{i}-u_{i-1}}\left(u-u_{i-1}\right), \quad u \in\left(u_{i-1}, u_{i}\right) .
\end{gathered}
$$

We now use these linear approximations to approximate $t_{i}$ :

$$
\begin{aligned}
t_{i} & =t_{i-1}+\left(u_{i}-u_{i-1}\right)+\int_{u_{i-1}}^{u_{i}} \frac{2 \frac{d b}{d u}}{1+\sqrt{1-F(b(u))}} d u \\
& \approx t_{i-1}+\left(u_{i}-u_{i-1}\right)+\int_{u_{i-1}}^{u_{i}} \frac{2 \frac{b_{i}-b_{i-1}}{u_{i}-u_{i-1}}}{1+\sqrt{1-F_{i-1}-\frac{F_{i}-F_{i-1}}{u_{i}-u_{i-1}}\left(u-u_{i-1}\right)}} d u \\
& =t_{i-1}+\left(u_{i}-u_{i-1}\right)+\frac{4\left(b_{i}-b_{i-1}\right)}{F_{i}-F_{i-1}}\left[\log \left(\frac{1+w_{i}}{1+w_{i-1}}\right)-\left(w_{i}-w_{i-1}\right)\right] .
\end{aligned}
$$

Substituting this approximation for $t_{i}$ into the first governing equation, (17a), yields a discretized version of the first governing equation:

$$
\begin{aligned}
u_{i+k}=\left(\frac{\alpha-\beta}{\alpha}\right)\{ & \frac{1-b_{i}}{w_{i}}+t_{i-1}+\left(u_{i}-u_{i-1}\right) \\
& \left.+\frac{4\left(b_{i}-b_{i-1}\right)}{F_{i}-F_{i-1}}\left[\log \left(\frac{1+w_{i}}{1+w_{i-1}}\right)-\left(w_{i}-w_{i-1}\right)\right]-1\right\}, \quad 1 \leq i \leq N f-k .
\end{aligned}
$$

\section{7 $\quad$ Iterative Procedure}

Using (18) we may determine all $F_{i}$ and $w_{i}$ values, for $1 \leq i \leq N_{f}$. Suppose, that for a given value of $i, i<N_{f}-k$, we know the values $b_{1}, \ldots, b_{i-1}, t_{1}, \ldots, t_{i-1}, u_{1}, \ldots, u_{i+k-1}$ and $A_{1}, \ldots, A_{i+k-1}$. Then the discretized versions of the first and third governing equations, (22) and (25), are a pair of linear equations in the unknown quantities $b_{i}$ and $u_{i+k}$, in terms of known quantities. We may solve these equations to obtain the values of $b_{i}$ and $u_{i+k}$, and then use these values in (21) and (24) to determine $A_{i+k}$ and $t_{i}$. This procedure may be iterated until $i=N f-k$. Specifically, at each step calculate the quantities $Q, Q_{1}, \ldots, Q_{4}$ in 
terms of the known quantities:

$$
\begin{gathered}
Q=\frac{4}{F_{i}-F_{i-1}}\left[\log \left(\frac{1+w_{i}}{1+w_{i-1}}\right)-\left(w_{i}-w_{i-1}\right)\right] \\
Q_{1}=u_{i+k-1}+\frac{2}{F_{i+k}+F_{i+k-1}}\left[\frac{\left(1-w_{i}\right)^{2}}{w_{i}}-A_{i+k-1}+A_{i}\right] \\
Q_{2}=\frac{2\left(1-w_{i}\right)^{2}}{w_{i}\left(F_{i+k}+F_{i+k-1}\right)} \\
Q_{3}=\left(\frac{\alpha-\beta}{\alpha}\right)\left[\frac{1}{w_{i}}+t_{i-1}+\left(u_{i}-u_{i-1}\right)-Q b_{i-1}-1\right] \\
Q_{4}=\left(\frac{\alpha-\beta}{\alpha}\right)\left[-\frac{1}{w_{i}}+Q\right]
\end{gathered}
$$

The updated values may be calculated as follows:

$$
\begin{aligned}
b_{i}=\frac{Q_{1}-Q_{3}}{Q_{2}+Q_{4}} & t_{i}=t_{i-1}+\left(u_{i}-u_{i-1}\right)+\left(b_{i}-b_{i-1}\right) Q \\
u_{i+k}=Q_{1}-b_{i} Q_{2} & A_{i+k}=A_{i}+\frac{\left(1-w_{i}\right)^{2}}{w_{i}}\left(1-b_{i}\right)
\end{aligned}
$$

(26) and (27) completely summarize the core of our iterative procedure. Specifically, given the initial seed values $b_{1}, t_{1}, u_{1}, \ldots, u_{1+k}$ and $A_{1}, \ldots, A_{1+k}$, we iteratively use (26) and (27) to determine $b_{1}, \ldots, b_{N_{f}-k}, t_{1}, \ldots, t_{N_{f}-k}, u_{1}, \ldots, u_{N_{f}}$ and $A_{1}, \ldots, A_{N_{f}}$. At the conclusion of this procedure, the only undetermined quantities will be the $\left(b_{i}, t_{i}\right)$ values in the last segment, from $i=N_{f}-k+1$ to $i=N_{f}$.

\subsection{Initializing Seed Values}

To initiate the above iterative procedure, we must provide values for $b_{1}, t_{1}, u_{1}, \ldots, u_{1+k}$ and $A_{1}, \ldots, A_{1+k}$. If we choose $N$ large enough, then $F_{1}, \ldots, F_{1+k}$ will be close to 0 , and over the interval $\left(0, u_{1+k}\right)$ we can approximate $F(b(u))$ as a linear function of $u$. We apply the discretized versions of the first and third governing equations, which enable us to solve for $u_{1}$ and $b_{1}$, and, hence, determine all necessary initializing seed values. Specifically, suppose that over the interval $\left(0, u_{1+k}\right)$ we approximate $F(b(u)) \approx \frac{F_{1}}{u_{1}} u$. Therefore, for $i=1, \ldots, k+1$, $u_{i}=\frac{u_{1}}{F_{1}} F_{i}$. From the discretized version of the first governing equation, (25),

$$
u_{1+k}=\left(\frac{\alpha-\beta}{\alpha}\right)\left\{\frac{1-b_{1}}{w_{1}}+u_{1}+\frac{4 b_{1}}{F_{1}}\left[\log \left(\frac{1+w_{1}}{2}\right)-\left(w_{1}-1\right)\right]-1\right\} .
$$


If we replace $u_{1+k}$ with $\frac{u_{1}}{F_{1}} F_{1+k}$ and recall that $\frac{F_{1+k}}{F_{1}}=\frac{\alpha}{\alpha-\beta}$, then after some algebraic simplification we obtain

$$
u_{1}\left[\left(\frac{\alpha}{\alpha-\beta}\right)^{2}-1\right]=\frac{1-w_{1}}{w_{1}}+\left[\frac{4}{F_{1}}\left\{\log \left(\frac{1+w_{1}}{2}\right)-w_{1}+1\right\}-\frac{1}{w_{1}}\right] b_{1} .
$$

Based on our linear approximation for $F(b(u))$ over the interval $\left(0, u_{1+k}\right)$, we may calculate the cumulative flow on this interval as

$$
A(u)=\int_{0}^{u} F\left(b\left(u^{\prime}\right)\right) d u^{\prime}=\frac{F_{1}}{u_{1}} \frac{u^{2}}{2} \quad u \in\left(0, u_{1+k}\right) .
$$

In particular, for $i=1, \ldots, 1+k, A_{i}=\frac{u_{1}}{2 F_{1}} F_{i}^{2}$. From the third governing equation, (19),

$$
A_{1+k}-A_{1}=\frac{\left(1-w_{1}\right)^{2}}{w_{1}}\left(1-b_{1}\right) .
$$

Replacing $A_{i+k}$ with $\frac{u_{1}}{2 F_{1}} F_{1+k}^{2}$ and $A_{1}$ with $\frac{u_{1} F_{1}}{2}$, and recalling that $\frac{F_{1+k}}{F_{1}}=\frac{\alpha}{\alpha-\beta}$, we obtain

$$
\frac{u_{1} F_{1}}{2}\left[\left(\frac{\alpha}{\alpha-\beta}\right)^{2}-1\right]=\frac{\left(1-w_{1}\right)^{2}}{w_{1}}\left(1-b_{1}\right) .
$$

(28) and (29) are a pair of linear equations which may be solved to obtain $u_{1}$ and $b_{1}$. Since $u_{i}=\frac{u_{1}}{F_{1}} F_{i}$ for $i=1, \ldots, 1+k$, we may determine $u_{2}, \ldots, u_{1+k}$. Since our linear approximation implies that $A_{i}=\frac{F_{1}}{u_{1}} \frac{u_{i}^{2}}{2}$ for $i=1, \ldots, 1+k$, we may determine $A_{1}, \ldots, A_{1+k}$. Finally, using (24) we may determine $t_{1}$. To summarize,

$$
\begin{gathered}
b_{1}=\frac{\left(1-w_{1}\right)\left(1-w_{1}-\frac{F_{1}}{2}\right)}{2 w_{1} \log \left(\frac{1+w_{1}}{2}\right)+1-w_{1}^{2}-\frac{F_{1}}{2}} \\
u_{1}=\frac{2\left(1-w_{1}\right)^{2}\left(1-b_{1}\right)}{F_{1} w_{1}\left[\left(\frac{\alpha}{\alpha-\beta}\right)^{2}-1\right]} \\
t_{1}=u_{1}+\frac{4 b_{1}}{F_{1}}\left[\log \left(\frac{1+w_{1}}{2}\right)-w_{1}+1\right] \\
u_{i}=\frac{u_{1}}{F_{1}} F_{i}, \quad i=2, \ldots, 1+k \\
A_{i}=\frac{u_{i}}{2 F_{1}} F_{i}^{2}, \quad i=1, \ldots, 1+k .
\end{gathered}
$$




\subsection{Final Segment}

After implementing the above iterative procedure, we will have determined all $F_{i}, w_{i}, u_{i}$ and $A_{i}$ values for $i=1, \ldots, N_{f}$, and will have determined all $b_{i}, t_{i}$ values for $i=1, \ldots, N_{f}-k$. The only remaining values to determine are $\left(b_{N_{f}-k+1}, t_{N_{f}-k+1}\right), \ldots,\left(b_{N_{f}}, t_{N_{f}}\right)$, corresponding to the $(x, t)$ coordinates of the lower boundary curve in the final segment. Furthermore, since we have determined $u_{N_{f}}$ (the departure time at $x=0$ of the final cohort of vehicles which arrives at the CBD exactly at time $\bar{t}$ ), from (4) we can calculate $\bar{t}$ as $\bar{t}=\frac{\alpha}{\alpha-\beta} u_{N_{f}}+1$.

In constructing the final segment, the first governing equation will no longer be applicable, since the characteristic lines emanating from the final segment will intersect the CBD at a point greater than $\bar{t}$, which does not contain the intersection of any vehicle trajectories. Recall that in deriving the third governing equation, we first determined the change in the cumulative flow along a characteristic line from the lower boundary of the departure set up to a vehicle trajectory (16). We simplified this equation by only considering the point where the vehicle trajectory intersects the CBD, i.e., by setting $u=u_{f}$ so that $\tilde{x}(u)=\bar{x}$ in (16), yielding the third governing equation. Here we use (16) in its more general form to determine the final segment of the lower boundary curve.

Suppose that $\left(b_{i}, t_{i}\right)$ is known for some $N_{f}-k \leq i<N_{f}$, and we wish to determine $\left(b_{i+1}, t_{i+1}\right)$. Subdivide the flow from its value $F_{i}$ at $\left(b_{i}, t_{i}\right)$ to its value $F_{i+1}$ at $\left(b_{i+1}, t_{i+1}\right)$ into $m$ equal values, and let $\left(F_{i}\right)_{j}$ denote the flow value at the $j$ th subdivision, so $\left(F_{i}\right)_{j}=$ $F_{i}+\frac{j}{m}\left(F_{i+1}-F_{i}\right), j=0, \ldots, m$. Based on these values we may calculate $\left(w_{i}\right)_{j}=\sqrt{1-\left(F_{i}\right)_{j}}$ and $\left(v_{i}\right)_{j}=\frac{1}{2}\left(1+\sqrt{1-\left(F_{i}\right)_{j}}\right)$, for $j=0, \ldots, m$. If we use the linear approximations for $F(b(u))$ and $b(u),(23)$, and the resulting time coordinate along the lower boundary curve, $(24)$, then we may approximate the space-time coordinates along the lower boundary curve corresponding to the sequence with $j=0, \ldots, m$ as $\left(\left(b_{i}\right)_{j},\left(t_{i}\right)_{j}\right)$, where

$$
\begin{gathered}
\left(b_{i}\right)_{j}=b_{i}+\frac{j}{m}\left(b_{i+1}-b_{i}\right) \\
\left(t_{i}\right)_{j}=t_{i}+\frac{j}{m}\left(u_{i+1}-u_{i}\right)+\frac{4\left(b_{i+1}-b_{i}\right)}{F_{i+1}-F_{i}}\left[\log \left(\frac{1+\left(w_{i}\right)_{j}}{1+w_{i}}\right)-\left\{\left(w_{i}\right)_{j}-w_{i}\right\}\right] .
\end{gathered}
$$

Consider the vehicle trajectory which passes through the lower boundary at $\left(b_{i+1}, t_{i+1}\right)$, and denote its space-time coordinates in Region II as $(\tilde{x}, \tilde{t})$. The characteristic line emanating from $\left(\left(b_{i}\right)_{j},\left(t_{i}\right)_{j}\right)$ intersects this vehicle trajectory at the point $\left(\tilde{x}_{j}, \tilde{t}_{j}\right), j=0, \ldots, m$. Beginning with $j=0$, proceed as follows. Approximate the trajectory curve through the point $\left(\tilde{x}_{j}, \tilde{t}_{j}\right)$ as a straight line with slope $\frac{d t}{d x}=\frac{1}{\left(V_{i}\right)_{j}}$, whose equation is given by

$$
x-\tilde{x}_{j}=\left(V_{i}\right)_{j}\left(t-\tilde{t}_{j}\right) .
$$

Now consider the characteristic line emanating from $\left(\left(b_{i}\right)_{j+1},\left(t_{i}\right)_{j+1}\right)$, which has slope $\frac{d t}{d x}=$ $\frac{1}{\left(w_{i}\right)_{j+1}}$, and whose equation is given by

$$
x-\left(b_{i}\right)_{j+1}=\left(w_{i}\right)_{j+1}\left(t-\left(t_{i}\right)_{j+1}\right) .
$$

The $(x, t)$ intersection of these two lines is taken as the $(j+1)$ st point along the vehicle 
trajectory, $\left(\tilde{x}_{j+1}, \tilde{t}_{j+1}\right)$, i.e.,

$$
\begin{gathered}
\tilde{t}_{j+1}=\frac{\left(w_{i}\right)_{j+1}\left(t_{i}\right)_{j+1}-\left(b_{i}\right)_{j+1}+\tilde{x}_{j}-\left(V_{i}\right)_{j} \tilde{t}_{j}}{\left(w_{i}\right)_{j+1}-\left(V_{i}\right)_{j}} \\
\tilde{x}_{j+1}=\left(w_{i}\right)_{j+1}\left(\tilde{t}_{j+1}-\left(t_{i}\right)_{j+1}\right)+\left(b_{i}\right)_{j+1} .
\end{gathered}
$$

If $m$ is chosen large enough, and we iterate this procedure from $j=0, \ldots, m-1$, then the final point on our trajectory, $\left(\tilde{x}_{m}, \tilde{t}_{m}\right)$, should coincide with the point on the lower boundary curve, $\left(b_{i+1}, t_{i+1}\right)$. The above procedure depends upon our initial choice for $b_{i+1}$, and the magnitude of our error in choosing $b_{i+1}$ can be measured by the difference between $\tilde{x}_{m}$ and $b_{i+1}$. Thus, to determine $b_{i+1}$, we construct a function which calculates this error for various values of $b_{i+1}$, and choose the value of $b_{i+1}$ which minimizes this error. Once we have determined $b_{i+1}$ we update $t_{i+1}$, and then iteratively repeat this procedure until obtaining $\left(b_{N_{f}}, t_{N_{f}}\right)$. A graph illustrating these concepts is provided in Figure 11.

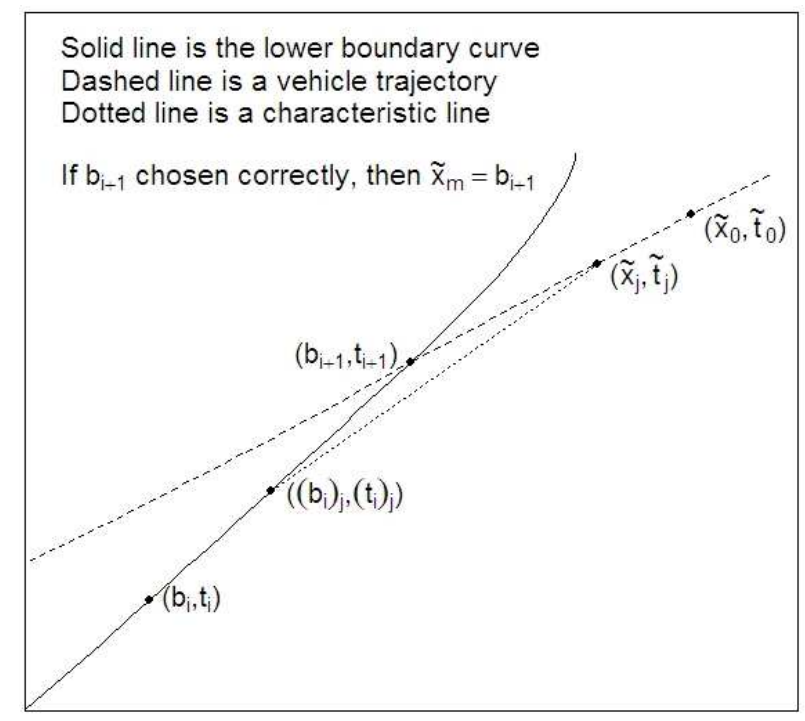

Figure 11: For $i=N_{f}-k+1$ to $i=N_{f}-1$, proceed as follows. Guess a value of $b_{i+1}$, and subdivide the lower boundary curve from $b_{i}$ to $b_{i+1}$ into $m$ segments, $\left(b_{i}\right)_{0}=$ $b_{i}, \ldots,\left(b_{i}\right)_{j}, \ldots,\left(b_{i}\right)_{\tilde{m}}=b_{i+1}$. Iteratively construct the vehicle trajectory through $\left(b_{i+1}, t_{i+1}\right)$ from the point $\left(\tilde{x}_{0}, \tilde{t}_{0}\right)$ to $\left(\tilde{x}_{m}, \tilde{t}_{m}\right)$. If $b_{i+1}$ was chosen correctly, then $\tilde{x}_{m}=b_{i+1}$.

\subsection{Summary of Numerical Solution}

To solve for the lower boundary curve and the flow along the lower boundary curve, we discretize the lower boundary curve into $N_{f}=N k$ pieces and assume that on each piece the $x$-coordinate of the lower boundary curve, $b(u)$, and also the flow on the lower boundary curve, $F(b(u)$ ), can both be approximated as linear functions of $u$. Note that at the beginning of the numerical procedure we will have already determined the flow values at all points on the lower boundary curve, i.e., we will have already determined $F_{i}$ for $i=1, \ldots, N_{f}$, and the remaining values which must be determined are the corresponding $u_{i}$ and $b_{i} \equiv b\left(u_{i}\right)$ 
values. The linear approximations for $b(u)$ and $F(b(u))$ on each piece of the lower boundary curve will be valid provided that our discretization is fine enough. Based on the linear approximation for the first segment of the lower boundary curve, we uniquely determine the initializing seed values for the iterative procedure, $b_{1}, u_{1}, \ldots, u_{1+k}, t_{1}, A_{1}, \ldots, A_{1+k}$, as in (30).

The iterative procedure relies only upon the linear approximations for $b(u)$ and $F(b(u))$ on each piece of the lower boundary curve, and uses the values $b_{1}, \ldots, b_{i-1}, u_{1}, \ldots, u_{i+k-1}$, $t_{1}, \ldots, t_{i-1}, A_{1}, \ldots, A_{i+k-1}$ to uniquely determine the values $b_{i}, u_{i+k}, t_{i}, A_{i+k}$ as in (26) and (27). The procedure stops after determining the solution up to the last segment, i.e., up to the last $k$ pieces of the lower boundary curve. At this stage all $u_{i}, A_{i}$ values will have been determined, $i=1, \ldots, N_{f}$, and the only values which remain to be determined are the $b_{i}, t_{i}$ values for $i=N_{f}-k+1, \ldots, N_{f}$.

To construct this final segment of $k$ pieces, given a known value of $b_{i}$ on one piece we guess a value for $b_{i+1}$ on the next piece. We then further subdivide this single piece into $m$ subdivisions. The characteristic lines emanating from the endpoints of these $m$ subdivisions partition the vehicle trajectory curve through $b_{i+1}$ into $m$ pieces. We assume that, over each of the $m$ partitions, the vehicle trajectory curve can be approximated by a linear function, which will be valid if the subdivisions are chosen fine enough, i.e., if $m$ is chosen large enough. We then use this linear approximation for the vehicle trajectory curve on each subdivision to explicitly calculate the vehicle trajectory curve. If our initial guess for $b_{i+1}$ was correct, then the vehicle trajectory curve we calculate should intersect the lower boundary curve at $b_{i+1}$. We try different values of $b_{i+1}$ until obtaining one such that the vehicle trajectory curve we calculate based on the $b_{i+1}$ value intersects the lower boundary curve at $b_{i+1}$ with sufficiently small error. Using this $b_{i+1}$ value we calculate $t_{i+1}$ based on our linear approximation for $b(u)$ on the $i+1$ th piece. This procedure is iterated until all $b_{i}, t_{i}$ values have been determined.

Provided that $N$ and $k$ are chosen large enough, our numerical procedure is based on valid linear approximations. Furthermore, the numerical procedure uniquely constructs a solution for the lower boundary curve and the flow along the curve, reaching capacity flow at the tip of the departure set. Thus, our numerical procedure provides a constructive proof that, using Greenshields' relation and given a ratio of parameter values $\frac{\beta}{\alpha}$, there is a unique solution to the Corridor Problem with the modified departure set that reaches capacity flow, i.e., there is a unique solution to the Corridor Problem that does not include mass departure points along the lower boundary of the departure set that reaches capacity flow.

We restate the comments from the beginning of this section, that the existence of a departure set solution which reaches capacity flow implies a continuous family of truncated solutions which do not reach capacity flow. Thus, using Greenshields' relation and given $\frac{\beta}{\alpha}$, for any flow value which is less than or equal to capacity flow, there is a unique solution to the Corridor Problem with the modified departure set that reaches this flow value at the tip of its departure set. 


\section{Numerical Results (Greenshields)}

\subsection{Departure Set Solutions}

We implement the numerical procedure for the ratio of parameter values $\frac{\beta}{\alpha}=0.2,0.4,0.6$ and 0.8. A graph of the departure sets, along with the vehicle trajectory corresponding to the final cohort of vehicles, is displayed in Figure 12. We have plotted all graphs with the same axes, to discern the behaviour of the solution with respect to the ratio of parameter values. As the unit time early cost, $\beta$, approaches the unit travel time cost, $\alpha$, the width and length of the departure set decreases, approaching free-flow condition of zero traffic departing $x=0$ at time $u=0$ and arriving at the CBD at time $\bar{t}=1$. In each of these graphs the flow reaches the capacity flow value of 1 at the tip of the departure set. At this point the slope of the characteristic curves will be infinite, and since the slope of the lower boundary curve must be greater than the slope of the characteristic curves, it must also be infinite. We observe this behaviour in our numerical solutions, as the tip of the departure set becomes vertical.
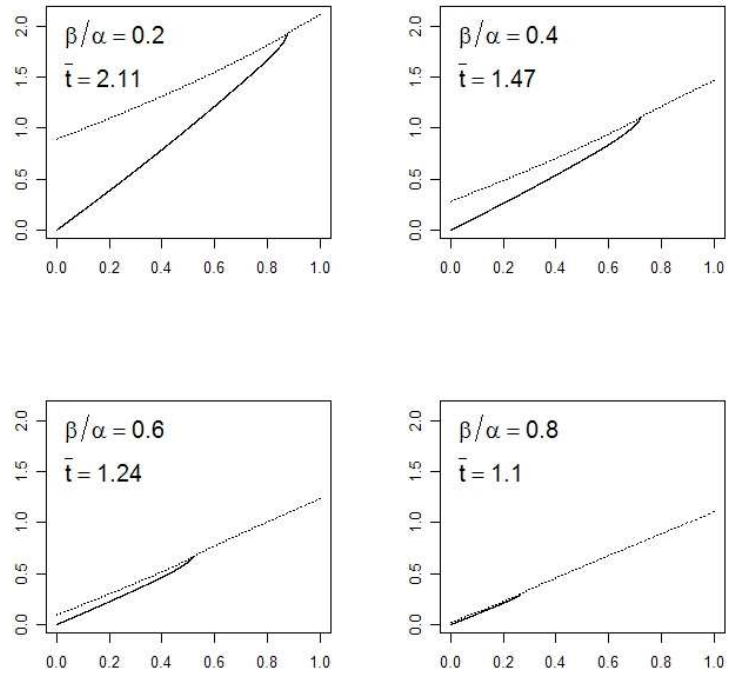

Figure 12: Numerically constructed departure set solutions for various ratios of parameter values, $0<\frac{\beta}{\alpha}<1$. The lower boundary curve of the departure set is graphed with a solid line. The upper boundary curve of the departure set, which corresponds to the trajectory of the final cohort of vehicles (which arrives at the CBD at $\bar{t}$ ), is graphed with a dashed line.

The following table lists the numerical values (to two decimal places) of several important features of our numerical solutions. 


\begin{tabular}{|l|cccc|}
\hline \multicolumn{4}{|c|}{ Numerical Results with $\bar{x}=1, V_{0}=1$ and $\rho_{J}=4$} \\
\hline & 0.2 & 0.4 & 0.6 & 0.8 \\
\hline \hline $\begin{array}{l}\text { Width of Departure Set at } x=0 \\
\text { (time units) }\end{array}$ & 0.89 & 0.28 & 0.10 & 0.02 \\
\hline $\begin{array}{l}\text { Tip of Departure Set } \\
\text { (distance, time) units }\end{array}$ & $(0.88,1.93)$ & $(0.72,1.10)$ & $(0.52,0.67)$ & $(0.27,0.31)$ \\
\hline $\bar{t}$ (time units) & 2.11 & 1.47 & 1.24 & 1.10 \\
\hline $\begin{array}{l}\text { Total Population } \\
\text { (population units) }\end{array}$ & 0.43 & 0.13 & 0.03 & 0.005 \\
\hline
\end{tabular}

\subsection{Corresponding Population Densities}

We previously showed how the TT condition implies that, at each location, within the interior of the departure set and on the upper boundary of the departure set the departure rate, $n(x, t)$, is constant $(9)$. We made the additional assumption that there are no mass departures on the lower boundary of the departure set, so that the departure rate is also constant over the lower boundary of the departure set. Hence, once we have numerically determined the flow, we may numerically differentiate (10) to determine the constant departure rate at each location. From (2), we may determine the population density at each location by multiplying the departure rate at that location by the width of the departure set at that location.

In Figure 13 we have graphed the population densities corresponding to the sample departure set solutions in Figure 12. The integral of the population density is the total population, which also equals the cumulative flow value along the trajectory in Region II for the final cohort of vehicles. Note that if we considered a truncated departure set solution which did not reach the capacity flow value of 1 , then the corresponding population densities would have the same general shape but would include less overall population and would reach zero earlier.

\subsection{Interpretation of Results}

To gain a more intuitive feeling for our results, we transform our results using more realistic values of $\bar{x}, V_{0}$ and $\rho_{J}$. Suppose the residential settlement is $\bar{x}=10 \mathrm{mi}$ long. To satisfy $\bar{x}=1$ we must choose distance units so that

$$
1 \text { distance unit }=10 \mathrm{mi} \text {. }
$$

Suppose free-flow velocity is $V_{0}=50 \mathrm{mi} / \mathrm{hr}$. To satisfy $V_{0}=1$ we must choose time units so that

$$
50 \mathrm{mi} / \mathrm{hr}=\frac{10 \mathrm{mi}}{\frac{1}{5} \mathrm{hr}}=\frac{1 \text { distance unit }}{\frac{1}{5} \mathrm{hr}}=1 .
$$

Thus,

$$
1 \text { time unit }=\frac{1}{5} \mathrm{hr}=12 \mathrm{~min}
$$



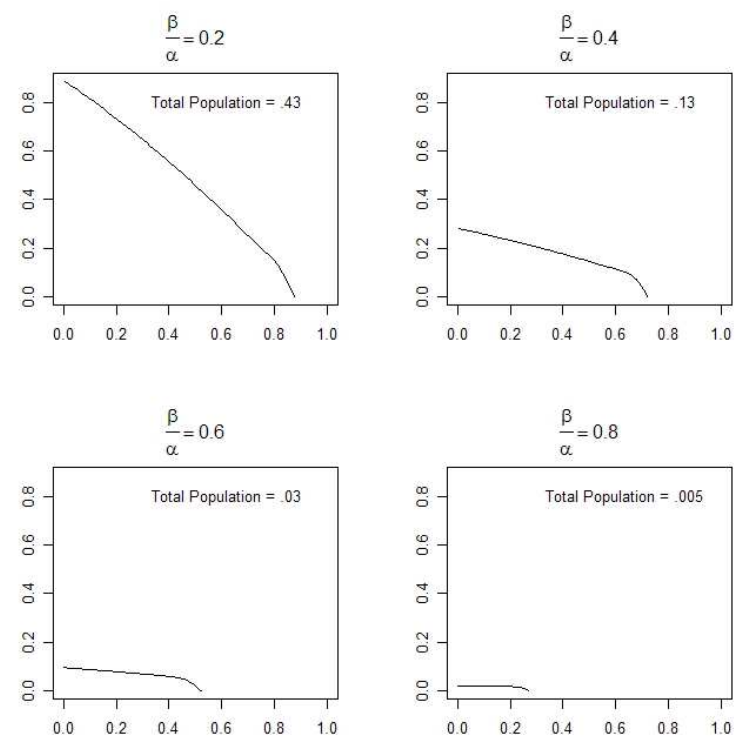

Figure 13: Population densities corresponding to the departure set solutions in Figure 12. We calculated the departure rate at each location by numerically differentiating the flow values with respect to location. The population density is obtained by multiplying the departure rate by the departure set width at that location.

Suppose the jam-density for a single traffic lane is $\frac{1 \text { vehicle }}{16 \mathrm{ft}}$. If the road has a constant width of four lanes, then the jam density of the road is $\frac{4 \text { vehicles }}{16 \mathrm{ft}}$. To satisfy $\rho_{J}=4$ we must choose population units so that

$$
\frac{4 \text { vehicles }}{16 \mathrm{ft}}=1320 \frac{\text { vehicles }}{\mathrm{mi}}=\frac{13,200 \text { vehicles }}{10 \mathrm{mi}}=4 \times \frac{3300 \text { vehicles }}{1 \text { distance unit }}=4 .
$$

Thus,

1 population unit $=3300$ vehicles.

To put this number into perspective, let us suppose that the city is 4 miles wide and that $5 \%$ of the city area is used for roads from the suburbs to the CBD. Since the typical lane width is 11 feet, there would be $[(5280)(5)(0.05)] \div[(4)(11)]=30$ such roads in the city, in which case a population unit for the entire city would be 99,000 vehicles.

For each of the four ratios of parameters $\frac{\beta}{\alpha}=0.2,0.4,0.6,0.8$, the departure set solutions and corresponding population densities have the same graphs as shown in Figures 12 and 13, except that we must use the above units of distance, time and population along the axes in those figures. The following table lists the numerical values of the same features presented in the previous table, but using the current system of units. 


\begin{tabular}{|l|cccc|}
\hline \multicolumn{5}{|c|}{ Numerical Results with $\bar{x}=10 \mathrm{mi}, V_{0}=50 \mathrm{mi} / \mathrm{hr}$ and $\rho_{J}=\frac{4 \text { vehicles }}{16 \mathrm{ft}}$} \\
\hline & 0.2 & 0.4 & 0.6 & 0.8 \\
\hline \hline $\begin{array}{l}\text { Width of Departure Set at } x=0 \\
\text { (minutes) }\end{array}$ & 10.7 & 3.4 & 1.2 & 0.2 \\
\hline $\begin{array}{l}\text { Tip of Departure Set } \\
\text { (miles, minutes) }\end{array}$ & $(8.8,23.2)$ & $(7.2,13.2)$ & $(5.2,8.0)$ & $(2.7,3.7)$ \\
\hline $\bar{t}$ (minutes) & 25.3 & 17.6 & 14.9 & 13.2 \\
\hline $\begin{array}{l}\text { Total Population } \\
\text { (vehicles) }\end{array}$ & 1419 & 429 & 99 & 16.5 \\
\hline
\end{tabular}

\section{Concluding Remarks}

Determining the equilibrium traffic flow over the course of a day for an entire metropolitan area is an important unsolved problem in urban transport economic theory and in transportation science. The problem is important since capacity should be chosen to accommodate maximum daily flow, which static models do not predict. This paper has considered perhaps the simplest variant of the problem, in which there is a single traffic corridor connecting a continuum of residential locations to the central business district (CBD). There is an exogenous density of identical vehicles along the corridor, each of which makes a morning trip to the CBD with a common desired arrival time. The road is of constant width and there is classic flow congestion. A vehicle's trip price is linear in travel time and early arrival time (late arrivals are not permitted). Equilibrium satisfies the trip-timing condition that no vehicle can lower its trip price by altering its departure time. What is the equilibrium pattern of departures? We termed this problem and related extensions (such as the social optimum and the equilibrium with heterogeneous vehicles and price-sensitive demand), The Corridor Problem.

Even the simplest variant of The Corridor Problem outlined above appears very difficult to solve. We have not yet succeeded in obtaining a complete solution to the problem, and in this paper reported preliminary results. We started by deriving properties of the equilibrium departure set analytically:

- The departure set is connected, contains no holes, and (in $x$ - $t$ space) is horn shaped.

- At each location, in the interior of the departure set, density, velocity, flow, and the departure rate are constant; at more central locations, density and flow are higher and velocity is lower; and the upper boundary of the departure set is a vehicle trajectory (so that the last vehicle trajectory, which corresponds to on-time arrival, contains vehicles from all locations) and contains no mass points.

- These properties correspond to a situation where the only vehicles to travel in the earliest cohort are from the most distant location, and as time proceeds vehicles are added to the cohort at successively more central locations.

Making the additional assumption that there are no mass points along the lower boundary of the departure set, we constructively determined a unique departure pattern along 
the traffic corridor which reaches capacity flow for each ratio of $\frac{\beta}{\alpha}$ (the ratio of unit time early cost to unit travel time cost). This departure pattern, which reaches capacity flow, implies a continuous family of truncated departure patterns which do not reach capacity flow. Furthermore, each departure pattern uniquely determines a population density along the traffic corridor. We had originally hoped that we would be able to solve for the equilibrium departure pattern given any population density profile along the traffic corridor. That we were unable to do so suggests that we over-constrained the problem by imposing the assumption of no mass points along the lower boundary of the departure set. A natural conjecture is that mass points along the lower boundary of the departure set are a generic feature of equilibrium, and that the solution of the equilibrium departure set is possible for any population density profile along the corridor when mass points along the lower boundary of the departure set are admitted.

Once the basic no-toll corridor equilibrium problem is solved, there are numerous extensions that can be considered: the equilibrium with heterogeneous vehicles; the social optimum with identical and heterogeneous vehicles; decentralization of the social optimum via a time- and space-varying toll; non-uniform road width; first- and second-best (constrained by restrictions on the form of toll that may be applied) road capacity as a function of distance from the CBD; and the equilibrium and optimal allocation of land along the corridor. However, before any of these extensions can be undertaken, a full solution to the basic Corridor Problem is needed.

\section{References}

Arnott, R. (1979). Unpriced transport congestion. Journal of Economic Theory, 21:294-316.

Arnott, R. (2004). The corridor problem: No-toll equilibrium. Mimeo.

Arnott, R., dePalma, A., and Lindsey, R. (1994). Bottlenecks in series. Notes.

Beckmann, M. and Puu, T. (1985). Spatial Economics: Density, Potential and Flow. Studies in Regional Science and Urban Economics 14, Amsterdam: North-Holland.

Evans, L. (2002). Partial Differential Equations. American Mathematical Society, Providence, Rhode Island.

Kanemoto, Y. (1976). Cost-benefit analysis and the second-best use of land for transportation. Journal of Urban Economics, 4:483-503.

Newell, G. (1993). A simplified theory of kinematic waves in highway traffic, part 1: General theory. Transpn. Res.-B, 27B:281-287.

Rhee, H., Aris, R., and Amundson, N. (1986). First-Order Partial Differential Equations: Volume 1 Theory and Applications of Single Equations. Prentice-Hall, Englewood Cliffs, New Jersey.

Ross, S. and Yinger, J. (2000). Timing equilibria in an urban model with congestion. Journal of Urban Economics, 47:390-413. 
Small, K. (1982). The scheduling of consumer activities: Work trips. American Economic Review, 72:467-479.

Solow, R. (1972). Congestion, density, and the use of land in transportation. Swedish Journal of Economics, 74:161-173.

Solow, R. and Vickrey, W. (1971). Land use in a long, narrow city. Journal of Economic Theory, 3:430-447.

Tian, Q., Huang, H., and Yang, H. (2007). Equilibrium properties of the morning peak-period commuting in a many-to-one transit system. Transportation Research B: Methodological, 41:616-631.

Vickrey, W. (1969). Congestion theory and transport investment. American Economic Review, 59:251-260.

Yinger, J. (1993). Bumper to bumper: A new approach to congestion in an urban model. Journal of Urban Economics, 34:249-275. 


\section{Nomenclature}

\begin{tabular}{|c|c|}
\hline CBD & Central Business District \\
\hline $\mathrm{TT}$ & Trip-Timing condition \\
\hline$x$ & distance \\
\hline$t$ & time \\
\hline $\bar{x}$ & location of $\mathrm{CBD}$ \\
\hline $\bar{t}$ & work start time at the CBD \\
\hline$N(x)$ & population density \\
\hline$C$ & total travel cost \\
\hline$\alpha$ & unit cost of travel time \\
\hline$\beta$ & unit cost of time early arrival \\
\hline$T(x, t)$ & travel time from $(x, t)$ to the CBD \\
\hline$\rho(x, t)$ & density (vehicles/length) at $(x, t)$ \\
\hline$v(x, t)$ & velocity at $(x, t)$ \\
\hline$V(\rho)$ & velocity as a function of density \\
\hline$F$ & flow, density times velocity \\
\hline$n(x, t)$ & entry rate, or departure rate, onto the road at $(x, t)$ \\
\hline $\mathcal{D}$ & set of $(x, t)$ points at which departures occur \\
\hline$p(x)$ & equilibrium trip price of a departure at location $x$ \\
\hline$a$ & arrival time at the $\mathrm{CBD}$ \\
\hline$\hat{T}(x, a)$ & $\begin{array}{l}\text { travel time to the CBD of a departure from location } x \\
\text { and arriving at time } a\end{array}$ \\
\hline $\mathcal{A}$ & set of $(x, a)$ points for which arrival rate is positive \\
\hline$u$ & departure time of a vehicle departing location $x=0$ \\
\hline Region I & $(x, t)$ points within the departure set \\
\hline Region II & $(x, t)$ points below the departure set \\
\hline$b(u)$ & $\mathrm{x}$-coordinate of the lower boundary as a function of $u$ \\
\hline$T_{I}(u)$ & travel time to the lower boundary as a function of $u$ \\
\hline$A(u)$ & cumulative flow along the lower boundary \\
\hline$\hat{A}(x, t)$ & cumulative flow in Region II \\
\hline$u_{0}, u_{f}$ & pair of departure times from $x=0$, as in Figure 7 \\
\hline$(\tilde{x}, \tilde{t})$ & space-time coordinates of a vehicle trajectory in Region II \\
\hline$V_{0}$ & maximum, free-flow velocity (Greenshields') \\
\hline$\rho_{J}$ & jam density at which velocity is zero (Greenshields') \\
\hline$w$ & "wave-velocity" or slope of the flow-density curve, $\frac{d}{d \rho}(\rho V)$ \\
\hline$i, j$ & dummy indices \\
\hline$N$ & number of segment divisions of the lower boundary curve \\
\hline$k$ & number of subdivisions within each segment \\
\hline$N_{f}=N k$ & total number of points along the lower boundary curve \\
\hline$u_{i}, b_{i}, F_{i}, v_{i}, w_{i}, A_{i}, t_{i}$ & function values along the lower boundary curve \\
\hline$Q, Q_{1}, \ldots, Q_{4}$ & quantities calculated in the iterative procedure \\
\hline$m$ & $\begin{array}{l}\text { number of subintervals into which we divide each subdivision } \\
\text { when determining the final segment }\end{array}$ \\
\hline$\left(F_{i}\right)_{j},\left(w_{i}\right)_{j},\left(v_{i}\right)_{j},\left(b_{i}\right)_{j},\left(t_{i}\right)_{j}$ & $\begin{array}{l}\text { function values on the } j \text { th subinterval in the } i \text { th subdivision } \\
\text { of the final segment of the lower boundary curve }\end{array}$ \\
\hline
\end{tabular}




\section{Statement of Contribution/Potential Impact}

Vickrey's bottleneck model has improved our understanding of the dynamics of rush-hour traffic congestion. Though the model has been extended to very simple networks, it has not however provided much insight into the spatial dynamics of congestion within a metropolitan area during the rush hour. Understanding the spatial dynamics of congestion is important since at each location roads need to be designed to accommodate the maximum flow over the day. This paper looks at this problem in perhaps the simplest possible context: trip-timing equilibrium for the morning commute with identical commuters (except for location), a continuum of entry points and a corresponding population distribution along a traffic corridor, a common arrival location (the central business district), a common desired arrival time, a road of constant width, no late arrival, and no toll. The paper reports on progress made in solving this Corridor Problem. The first part of the paper demonstrates that in location-time space, the departure set is connected and "horn-shaped". The first cohort contains only vehicles departing from the metropolitan boundary; the last cohort contains vehicles departing from all locations; later cohorts contain vehicles from increasingly central locations, as well as from all more distant locations. The second part of the paper constructively determines the class of population distributions along the corridor consistent with the assumption that there are no mass points along the lower boundary of the departure set. That this class is restricted suggests that mass points along the lower boundary are a generic feature of the general solution. 